
Aus der Klinik für Augenheilkunde
Universitätsklinikum des Saarlandes
Homburg/Saar

Direktor: Prof. Dr. med. Berthold Seitz

\title{
Reproduzierbarkeit der Femtosekundenlaserschnitte bei der posterioren lamellären Keratoplastik in vitro
}

\author{
Dissertation \\ zur Erlangung des Grades eines Doktors der Medizin \\ der Medizinischen Fakultät \\ der UNIVERSITÄT DES SAARLANDES \\ 2011
}

vorgelegt von: Sebastian Harlfinger geb. am 28.02.1978 in Olpe 
Meiner Familie mit MB! 


\section{Inhaltsverzeichnis}

1 Zusammenfassung / Abstract .............................................................. 5

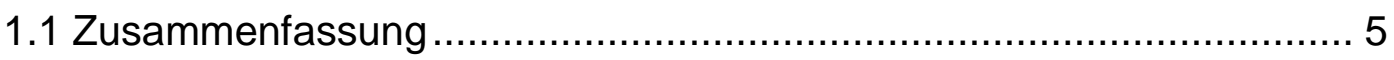

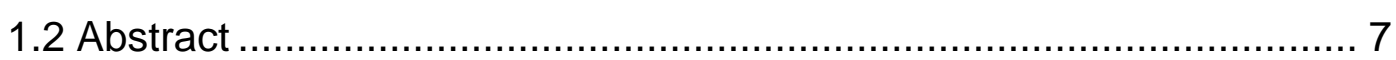

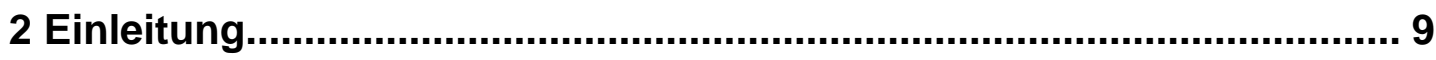

2.1 Methoden und Anwendungsgebiete der posterioren lamellären

Keratoplastik (PLAK) .......................................................... 9

2.1.1 Funktionsweise des Femtosekundenlasers .............................. 10

2.1.2 Die PLAK mittels des Femtosekundenlasers ........................... 12

2.2 Ziele und Fragestellungen der Studie........................................... 13

3 Material und Methoden .................................................................. 14

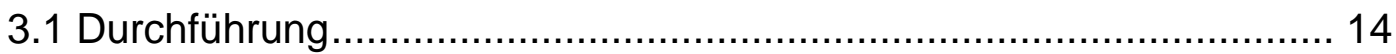

3.1.1 Laserprozedur................................................................ 14

3.1.2 Histologische Aufarbeitung und Beurteilung ............................ 14

3.1.3 Gemessene Parameter und Gruppeneinteilung ....................... 15

3.2 Statistische Methoden ............................................................ 19

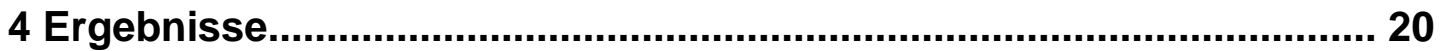

4.1 Intendierter Abstand der Bowman-Lamelle zur horizontalen Blasenlinie....................................................... 20

4.2 Anzahl der Blasen ................................................................... 24

4.3 Länge und Breite der Blasen .................................................. 27

4.4 Verhältnis Blasenlänge zur Länge der dazwischen liegenden Gewebestege .................................................................. 30

4.5 Zustand der Gewebestege zwischen den Blasen........................... 33

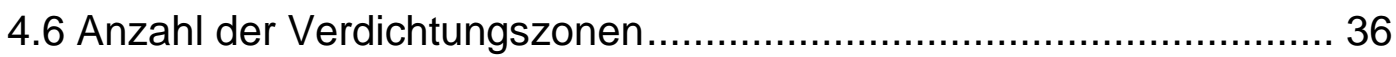

4.7 Zustand der Descemet-Membran ................................................ 37

4.8 Abstand der nächstliegenden Blasen zur Descemet-Membran.......... 39

4.9 Zusammenhang Abstand der nächstliegenden Blasen von der Descemet-Membran - Zustand der Membran ............................. 45

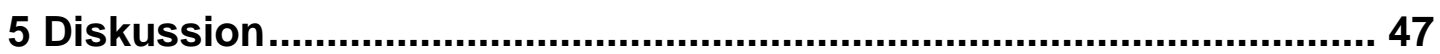

5.1 Abstand der Bowman-Lamelle zur horizontalen Blasenlinie .............. 47

5.2 Anzahl der Blasen ........................................................... 52

5.3 Länge und Breite der Blasen .............................................. 52

5.4 Verhältnis von Blasen zu Gewebestegen .................................... 53

5.5 Zustand des Gewebes und Verdichtungszonen ........................... 53 
5.6 Zusammenhang Abstand der nächstliegenden Blasen von der Descemet-Membran - Zustand der Membran

5.7 Darstellung der Verfahren

„Descemet Stripping Automated Endothelial Keratoplasty“ (DSAEK) und „Descemet Membrane Endothelial Keratoplasty“ (DMEK)........... 56

5.8 Vergleich der klinischen Ergebnisse

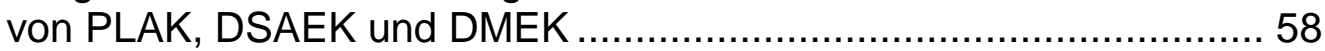

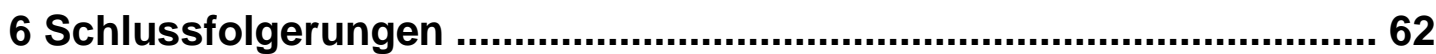

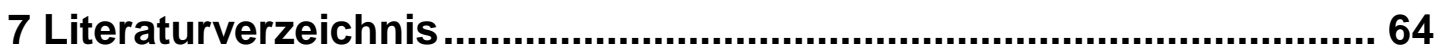

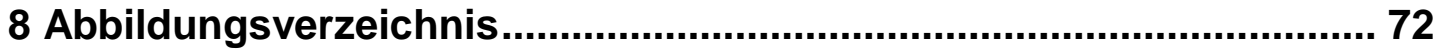

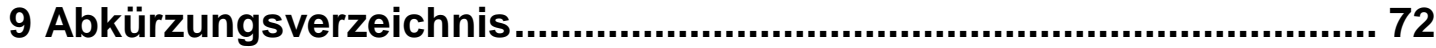

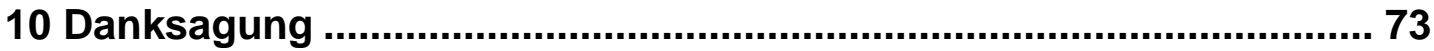




\section{Zusammenfassung / Abstract}

\subsection{Zusammenfassung}

\section{Hintergrund und Ziele}

Mit der anterioren Keratoplastik steht ein seit vielen Jahrzehnten angewandtes Verfahren zur Verfügung. Es gibt Bestrebungen, ähnliche Prinzipien der lamellären Chirurgie auch bei einer Technik anzuwenden, die die Integrität des vorderen Corneastromas bewahrt. Diese Technik wird unter anderem posteriore lamelläre Keratoplastik (PLAK) genannt.

Um einen Lentikel aus dem posterioren Teil des Stromas zu schneiden, kann ein Femtosekundenlaser verwendet werden. Die vorliegende Arbeit beschäftigt sich mit der Reproduzierbarkeit der Femtosekundenlaserschnitte bei dieser posterioren lamellären Keratoplastik.

\section{Material und Methoden}

Für die vorliegende Arbeit wurde der FEMTEC-Laser (20/10 Perfect Vision, Heidelberg, Deutschland) verwendet. Mittels dieses Lasers wurde eine posteriore lamelläre Dissektion durchgeführt. Die intendierten Abstände Bowman-Lamelle zu horizontaler Ebene des zu lasernden Lentikels wurden mit den gemessenen verglichen.

Nach der Laserprozedur erfolgten eine makroskopische Beurteilung sowie die histologische Aufarbeitung. Es wurde eine Gesamtzahl von 54 Schnitten analysiert. Die mikroskopische Auswertung wurde mit einem Lichtmikroskop (Olympus, BHB, Japan) durchgeführt.

\section{Ergebnisse}

1. Die Blasenanzahl nimmt mit zunehmendem Abstand des Lentikels von der Bowman-Lamelle in der vertikalen Ebene ab. In der horizontalen und vertikalen Ebene gibt es gleich viele Blasen. 
2. Der intendierte Abstand wird mit einer Abweichung zwischen 115-160 $\mu \mathrm{m}$ erreicht und ist gleichmäßig über die gesamte Länge des Lentikels.

3. Die Blasen in der Horizontalen sind im Vergleich zur Vertikalen länger. In der Vertikalen werden gleich lange und breite Blasen produziert.

4. In der horizontalen Ebene ist die Blasenlänge im Verhältnis zu den dazwischen liegenden Gewebestegen anteilig deutlich höher als in der Vertikalen.

5. Eine Verdichtungszone ist in $88,9 \%$ der Schnitte vorhanden.

6. In $63 \%$ der Schnitte findet sich eine Teilperforation oder Minimalläsion der Descemet-Membran.

7. Es gibt einen reproduzierbaren, gleichen Abstand der nächstliegenden Blasen zur Descemet-Membran.

8. Mit abnehmender Lentikeldicke steigt die Entfernung der beiden nächstliegenden Blasen zur Descemet-Membran.

\section{Schlussfolgerungen}

Mit dem Femtosekundenlaser existiert ein präzises Instrument für die Durchführung der posterioren lamellären Keratoplastik. Aufgrund der reproduzierbaren Anzahl von Blasen gleicher Größe ermöglicht er eine präzise Schnittführung. In ca. $2 / 3$ der Fälle ist eine Läsion der DescemetMembran nachweisbar, womit eine einfache Entfernung des entstandenen Lentikels möglich ist. Ein Nachteil ist die abnehmende Wahrscheinlichkeit einer Läsion der Descemet-Membran sowie der Trend einer abnehmenden Präzision bei geringerer Lentikeldicke. Relevante thermische oder durch Kompression entstandene Schäden wurden nicht gefunden.

Künftige Studien sollten sich auf die Entwicklung eines standardisierten Verfahrens mit optimierten Einstellungen zur Erreichung der Ziele (verlässliche Lentikeldicke, Perforation der Descemet-Membran) und Entwicklung einheitlicher Messverfahren zum besseren Vergleich konzentrieren. 


\subsection{Abstract}

Reproducibility of femtosecond laser dissections in the posterior lamellar keratoplasty in vitro

\section{Purpose}

For many decades, the anterior keratoplasty has been a standard procedure. For some time now, surgeons have tried to use this technique of keratoplasty in a way that keeps the anterior parts of the cornea intact. This technique is called posterior lamellar keratoplasty (PLAK). Femtosecond lasers can be used to cut a lenticle out of the posterior part of the stroma. This study deals with the reproducibility of the generated lenticles.

\section{Methods}

For this study the FEMTEC-Laser (20/10 Perfect Vision, Heidelberg, Germany) was used. With this laser we performed posterior lamellar dissections.

The goal was to assess the thickness of the graft in comparison to the intended thickness predefined with the laser. A macroscopic evaluation and histological preparation was done. A total amount of 54 corneas was analysed. For light microscopy we used an Olympus microscope (BHB, Japan).

\section{Results}

1. In the vertical layer, the number of bubbles declines with an increasing distance between lenticle and Bowman's layer. The number of bubbles is equal in the horizontal and vertical layers.

2. The intended distance is reached with a precision of about $115-160 \mu \mathrm{m}$ and is constant over the whole length of the lenticle. 
3. In comparison to the vertical layer, the bubbles in the horizontal layer are longer. On both vertical sides, equally long and wide bubbles may be found.

4. In the horizontal layer, the length of the bubbles compared to the tissue bridges inbetween them is higher.

5. At least one compaction zone could be found in $88.9 \%$ of the corneas.

6. $63 \%$ of the corneas show a partial perforation or minimal lesion of Descemet's membrane.

7. The distance of the two closest bubbles to Descemet's membrane is reproducible.

8. The distance of the two closest bubbles to Descemet's membrane increases in thinner lenticles.

\section{Conclusions}

The femtosecond laser proves to be an accurate tool for posterior lamellar keratoplasty. As the laser generates a reproductible number of bubbles of the same size, precise incisions can be achieved. In the majority of the cases a lesion of the Descemet's membrane could be found which seems to make the removal of the lenticles easier. A disadvantage is a decreasing probability of a lesion of the Descemet's membrane as well as the decreasing precision in thinner lenticles. Relevant damage that originated in compression or heat did not occur.

Future studies should focus on developing a standardized procedure with optimized settings in order to reach the desired goals (reliable thickness of lenticles, perforation of Descemet's membrane) as well as devise consistent measuring methods that enable to compare the results. 


\section{Einleitung}

\subsection{Methoden und Anwendungsgebiete der posterioren lamellären Keratoplastik (PLAK)}

Die Philosophie der lamellären Keratoplastik ist es, nur das krankhafte Gewebe zu ersetzen und das gesunde zu belassen [55]. Die anteriore Keratoplastik ist dabei seit mehr als einem Jahrhundert in Gebrauch [62]. Nachteile bzw. Komplikationen dieses Verfahrens sind entstehender Astigmatismus bzw. weitere Ametropien als mögliches Resultat der Wundheilung. Durch die Nähte kann es zu Infektionen, Ulzerationen und Neovaskularisationen kommen, welche $z u$ Abstoßungsreaktion führen können [57]. Seit einigen Jahren werden nun auch die Prinzipien der lamellären Chirurgie auf das posteriore Stroma und den Austausch des Endothels angewandt, d. h., das Spendergewebe wird in den posterioren Teil der Cornea eines Empfängers transplantiert. Häufige Indikationen dafür sind z. B. die Fuchssche endotheliale Hornhautdystrophie, die pseudophake bullöse Keratopathie und die Keratopathie beim Pseudoexfoliationssyndrom. Schätzungen gehen davon aus, dass bei ca. $40-45 \%$ von über 33.000 aller durchgeführten penetrierenden Keratoplastiken in den Vereinigten Staaten die Schäden der Cornea auf das Endothel und das posteriore Stroma beschränkt sind [15], d. h., das Anwendungsgebiet für die posteriore lamelläre Keratoplastik ist entsprechend groß. Die Vorteile, die man sich von ihr verspricht, liegen in der regulären Oberflächentopographie, der hohen Voraussagbarkeit der Brechkraft, der schnelleren visuellen Rehabilitation, der Klarheit der Cornea sowie einem stabilen Bulbus aufgrund des Erhalts der kompletten vorderen Stromalamelle, die für die mechanische Stabilität der Cornea verantwortlich ist $[58,61]$. Zudem soll durch die kleinere Inzision bei dieser Technik das Risiko von Infektionen, der expulsiven Blutung und Nahtkomplikationen verringert werden [13].

Der Vorteil einer operativen Technik wie der zuerst von MELLES et al. [33] vorgestellten posterioren lamellären Keratoplastik oder der von TERRY et al. [56] beschriebenen „DLEK“ (Deep Lamellar Endothelial Keratoplasty) liegt im Transfer gesunden Endothels, während die Integrität des vorderen 
Corneastromas des Empfängers eines Transplantates gewahrt bleibt. Ein möglicher Nachteil liegt hierbei jedoch in der schwierigen Operationstechnik und v. a. der schlechten Kontrolle über die zu transplantierende Lamellendicke sowohl beim Empfänger als auch beim Spender.

\subsubsection{Funktionsweise des Femtosekundenlasers}

Femtosekundenlaser sind meistens Infrarotlaser. Einige von innen erzeugen Laserimpulse mit einer Wellenlänge von ca. $1000 \mathrm{~nm}$, welche die transparente anteriore Schicht der Cornea passieren können, ohne Schäden zu erzeugen.

Ihre verstärkten, ultrakurzen Laserimpulse werden mit einer Wirkdauer von mehreren hundert Femtosekunden $\left(1 \mathrm{fs}=10^{-15} \mathrm{~s}\right.$ ) in den posterioren Anteil der Cornea fokussiert. Erst an diesem Fokuspunkt des Laserstrahls und nach Erreichen einer Schwellenfluenz, also einer bestimmten Energie pro Fläche, entsteht ein Mikroplasma, welches Stromagewebe verdampft. Dieser Prozess wird auch "laser induced optical breakdown" genannt [29]. Dadurch folgen sekundäre Effekte wie die Ausbreitung von Schockwellen und die Entstehung von Mikroblasen, welche sich ausdehnen und so die umgebenden Stromalamellen trennen [24]. Bei diesem - Photodisruption genannten - Vorgang entsteht ein Gemisch aus $\mathrm{CO}_{2}, \mathrm{~N}_{2}$ und $\mathrm{H}_{2} \mathrm{O}$, welches durch die endotheliale Pumpwirkung entfernt wird [23, 29].

Mit abnehmender Pulsdauer sinkt die Schwellenfluenz für diesen Prozess. Hierin liegt auch der Vorteil des Femtosekundenlasers gegenüber länger gepulsten Lasern. Da er eine geringere Pulsdauer aufweist, benötigt man bei seiner Verwendung insgesamt eine niedrigere Pulsenergie. Da biologisches Gewebe bei extrem kurzen Pulsen nicht reagiert, verringern sich auch die sekundären Effekte (Schockwellen, Mikroblasen) [23,29].

RATKAY-TRAUB et al. [44] geben z. B. an, dass ab 150 fs der Durchmesser der Kavitationsblase (= Mikroblase) ca. $3-15 \mu \mathrm{m}$ ist, bei $10 \mathrm{~ns}$ hingegen ca. 300-1200 $\mu \mathrm{m}$.

Fixiert man die gelaserten Corneae unmittelbar nach der Prozedur z. B. in Paraformaldehydlösung und bereitet sie für die histologische Beurteilung entsprechend auf, sind die entstandenen Mikroblasen im Stroma zu 
sehen - ohne diese Fixation würden sie nach ca. 10-15 Minuten verschwinden [30]. Größere Blasen können durch die Verschmelzung vieler einzelner durch benachbarte Laserpulse entstehen.

Im Gegensatz zu Lasern im sichtbaren Bereich des Spektrums ist der Femtosekundenlaser weniger anfällig für optische Trübungen, was einen Vorteil bei Schnitten in ödematösen Corneae darstellt, welche typischerweise bei endothelialen Dysfunktionen auftreten. Insgesamt scheint er also besonders geeignet für die PLAK zu sein [30].

Zurzeit gibt es vier verschiedene Femtosekundenlasersysteme: IntraLase FS (Einführung 2001), FEMTEC 20/10 Perfect Vision (Einführung 2001), Ziemer DaVinci (Einführung 2005) und Zeiss VisuMax (Einführung 2006).

Alle vier Systeme basieren auf dem beschriebenen Prinzip der Photodisruption. Der IntraLase sowie der FEMTEC 20/10 Perfect Vision arbeiten dabei mit einer hohen Pulsenergie und niedrigen Pulsfrequenz. Bei den beiden anderen ist es umgekehrt. Dies führt bei der ersten Gruppe zu vergleichsweise größeren Mikroblasen bei höherer Spotseparation - dies ist der Abstand zwischen zwei einwirkenden Laserimpulsen - wobei die Oberflächenrauheit der entstehenden Schnitte bei beiden Gruppen trotzdem nachweislich gleich ist [45]. Zur Fixierung der zu behandelnden Augen werden verschiedene Systeme verwendet. Das IntraLase System verwendet einen „suction ring“ mit einer flachen Glaskontaktlinse, der VisuMax und der FEMTEC 20/10 eine gewölbte Linse, wodurch diese beiden Systeme weniger Unterdruck zur Fixierung benötigen (ca. $-20 \mathrm{mmHg}$ im Vergleich zu -35 bis $-45 \mathrm{mmHg}$ beim IntraLase) und die Cornea nicht abflachen. Dies hat den Vorteil, dass sich die Cornea bei einem unvorhergesehenen Unterdruckverlust während der Laserprozedur nur wenig oder gar nicht verformt und der Schnitt ggf. wie vorgesehen ausgeführt werden kann [52]. Im Gegensatz zu den anderen drei Lasern kann der DaVinci Laser nur zur Produktion von Flaps und nicht für andere Zwecke wie z. B. lamelläre Keratoplastiken verwendet werden, da seine Fokusebene während des Schnittes nicht verändert werden kann.

Ein großer Vorteil des IntraLase-Systems ist die große klinische Erfahrung, die mittlerweile mit inm gesammelt werden konnte ( $>1$ Million behandelte Augen) [31]. 
Nachteil aller vier Systeme sind die strengen Anforderungen an die Umgebungstemperatur und die Luftfeuchtigkeit.

\subsubsection{Die PLAK mittels des Femtosekundenlasers}

Ziel einer automatisierten posterioren lamellären Keratoplastik unter Zuhilfenahme eines Femtosekundenlasers ist es, das Endothel, die Descemet-Membran und so wenig angrenzendes Stroma wie möglich herauszuschneiden. Um dies zu erreichen, wird mittels des Lasers ein Lentikel aus dem posterioren Anteil des Stromas geschnitten. Von SEITZ et al. wurde hierfür der Begriff Femto-PLAK vorgeschlagen [49].

Ein Vorteil des Femtosekundenlasers liegt darin, dass er in der Lage ist, gleichmäßige Lentikeldicken zu produzieren. Hingegen entstehen bei der Verwendung mechanischer Mikrokeratome lediglich meniskusartig geformte Lentikel [48].

JONAS konnte in einem Case Report zeigen, dass eine Prozedur möglich ist, bei der ein posteriorer Lentikel (bestehend aus Descemet-Membran, Endothel und einem schmalen Streifen Stroma) über einen anterioren Flap, der drei Spitzen sowie ein Scharnier aufweist (wie bei der LASIK-Prozedur), problemlos entnommen und wieder in einem analog behandelten EmpfängerAuge repositioniert werden kann. Die Dauer hierfür wurde mit unter 5 Minuten angegeben [21].

Im Jahr 2007 veröffentlichten HOFFART et al. einen Case Report, in dem sie von dem guten Erfolg einer "automated lamellar therapeutic keratoplasty“ berichteten. Hier wurden sowohl der Spender- als auch der Empfängerlentikel mittels des Femtosekundenlasers (20/10 Perfect Vision) erzeugt [18].

Auch ein erstes Transplantationsszenario, bei dem die Lamellen des Spendergewebes mittels des Lasers geschnitten werden und anschließend in Optisol-Lösung aufbewahrt werden, wurde bereits entwickelt [53].

Insgesamt lässt sich also für die klinische Anwendbarkeit folgern, dass mit Hilfe des zuverlässig arbeitenden Femtosekundenlasers ein schwieriger operativer Schritt vereinfacht werden kann [51]. 


\subsection{Ziele und Fragestellungen der Studie}

Ziel dieser Studie war der Nachweis der Reproduzierbarkeit der Femtosekundenlaserschnitte bei der posterioren lamellären Keratoplastik in vitro.

Hierzu wurden folgende Fragestellungen bearbeitet:

1. Inwieweit entspricht der gemessene Abstand zwischen Bowman-Lamelle und Lentikel dem vorher festgelegten? Bestehen innerhalb dieses Abstandes Unterschiede in den Streuungen der gemessenen Abstandswerte?

Existieren Streuungen in der Differenz zwischen gemessenem minimalem und maximalem Abstandswert von Bowman-Lamelle zum Lentikel?

2. Wie hoch ist die Anzahl der Blasen in der horizontalen und vertikalen Ebene?

Gibt es einen nachweislichen Zusammenhang zwischen dem Abstand der Bowman-Lamelle zum Lentikel einerseits und der Anzahl der Blasen andererseits - getrennt betrachtet nach vertikal links, vertikal rechts und horizontal?

3. Wie breit bzw. lang sind die Blasen, und gibt es einen überzufälligen Unterschied zwischen vertikaler und horizontaler Schnittebene?

4. Wie ist das Verhältnis der Länge der Gewebestege zu der Länge der Blasen und gibt es hierbei Unterschiede in der vertikalen und horizontalen Schnittebene?

Hat dieses Verhältnis einen Einfluss auf die Gewebebeschaffenheit der Stege?

5. Wie häufig und wie ausgeprägt kommen Verdichtungszonen vor?

6. Wie ist der Zustand der Descemet-Membran, ist sie z. B. unterbrochen? Wie ist der Abstand der beiden am nächsten liegenden Blasen zur Descemet-Membran, und existiert ein Zusammenhang zwischen diesem Abstand und dem Zustand der Membran? 


\section{Material und Methoden}

\subsection{Durchführung}

\subsubsection{Laserprozedur}

Für die vorliegende Arbeit wurde der FEMTEC-Laser (20/10 Perfect Vision, Heidelberg, Deutschland) verwendet. Dieser Laser arbeitete mit einer Wellenlänge von ca. $1 \mu \mathrm{m}$ bei einer Pulsenergie von $3,5 \mu \mathrm{J}$ für den horizontalen, lamellierenden und $6 \mu \mathrm{J}$ für den vertikalen trepanierenden Randschnitt bei einer Spotgröße von $<10 \mu \mathrm{m}$. Die Repetitionsrate betrug $12,5 \mathrm{kHz}$.

Der zu behandelnde Bulbus wurde vorsichtig fixiert. Als dreidimensionale Referenzposition diente eine gewölbte Kontaktlinse, die die gleiche Kurvatur wie das Auge aufwies und an einem Konus befestigt war, welcher Teil des „application arm“ des Lasers ist. Diese Linse wurde nun durch ein geringes Vakuum von ca. $-20 \mathrm{mmHg}$ auf der okularen Oberfläche fixiert, ohne diese abzuflachen. Dies stellt einen wesentlichen Unterschied zum in den Vereinigten Staaten weit verbreiteten IntraLase-System dar, bei dem der stabile Fokus durch corneale Applanation erzeugt wird (vgl. 2.1.1) [29, 63].

Ein Hochkontrast-Mikroskop war ebenfalls in die Einheit integriert.

Mit dem Laser wurde bei 60 frisch enukleierten Schweineaugen eine posteriore lamelläre Dissektion durchgeführt, die in der Vorderkammer begann und im hinteren Stroma endete (Dauer zwischen 31 und $90 \mathrm{~s}$ ).

Bei den Schweineaugen handelte es sich um am selben Tag frisch enukleierte, gebrühte Augen. Vor der Laserprozedur wurden die Augen einer Sichtprüfung unterzogen sowie manuell mit Hilfe eines Hockeymessers von evtl. vorhandenen Epithelresten befreit. Durch Fehler in der Behandlung nicht verwendbare Augen wurden noch vor der Laserprozedur verworfen.

\subsubsection{Histologische Aufarbeitung und Beurteilung}

Im direkten Anschluss an die Laserprozedur wurden die Bulbi in toto in $10 \%$ gepufferter Paraformaldehydlösung für $24 \mathrm{~h}$ fixiert. Danach wurden sie 
halbiert und beide Hälften in Paraffin eingebettet, auf $6 \mu \mathrm{m}$ Dicke geschnitten und nach Periodic Acid Schiff (PAS) gefärbt. Sechs Schnitte wurden durch diese Behandlung unbrauchbar und wurden nicht in die Arbeit einbezogen. Insgesamt wurde also jeweils ein Schnitt von 54 Bulbi analysiert.

Für die Auswertung mittels eines Lichtmikroskops (Olympus, BHB, Japan) wurden die Histologien in 20- bzw. 40-facher Vergrößerung mit einem Messokular betrachtet und vermessen.

\subsubsection{Gemessene Parameter und Gruppeneinteilung}

Für die Laserprozedur wurden insgesamt sechs Gruppen à zehn Augen gebildet, die sich jeweils nur durch den eingestellten Abstand BowmanLamelle - horizontale Ebene des zu lasernden Lentikels unterschieden.

Als Abstandswerte wurden eingestellt: $1000 \mu \mathrm{m}, 950 \mu \mathrm{m}, 850 \mu \mathrm{m}, 750 \mu \mathrm{m}$, $650 \mu \mathrm{m}$ und $550 \mu \mathrm{m}$.

Nach der histologischen Aufarbeitung wurden folgende Parameter gemessen bzw. beurteilt:

a. Abstand der Bowman-Lamelle und der Descemet-Membran zu den horizontalen bzw. vertikalen Blasen des entstandenen Lentikels

b. Anzahl der Blasen im Lentikel horizontal und vertikal

c. Länge und Breite der Blasen (die Länge entspricht der horizontalen, die Breite der vertikalen Ausdehnung)

d. Länge und Gewebebeschaffenheit der Gewebestege zwischen den Blasen (die Gewebestege wurden nach der Beschaffenheit der Stromalamellen beurteilt und es wurde zwischen den Gewebebeschaffenheiten „vakuolig“ (Abb. 1) und „aufgelockert" (Abb. 2) unterschieden) 


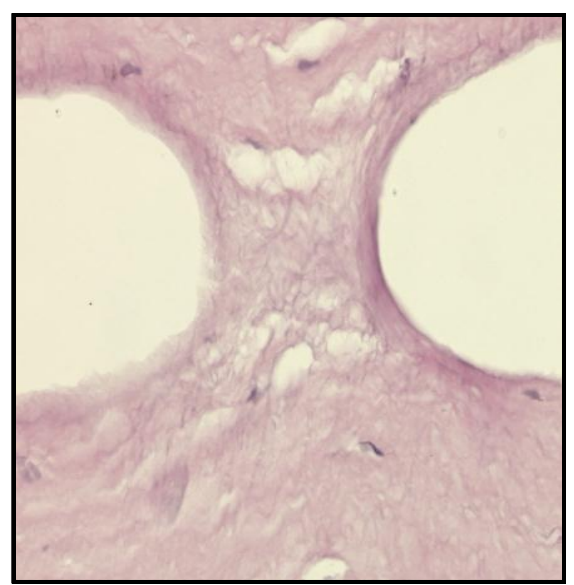

Abb. 1: „vakuolig“6 (x 20)

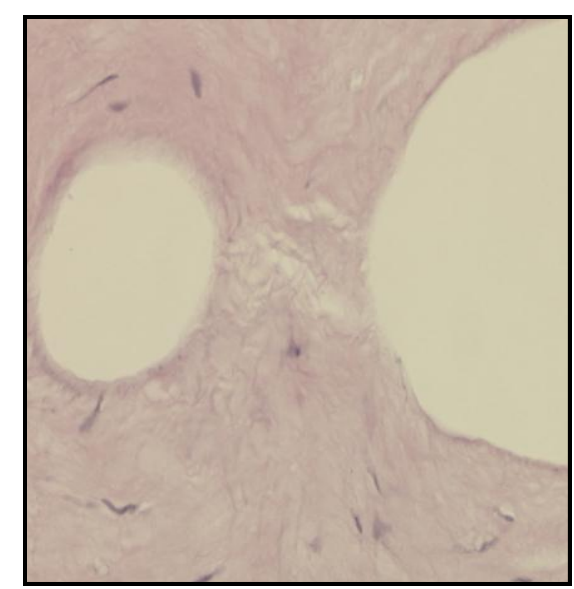

Abb. 2: „aufgelockert““ (x 20)

e. Zustand der Descemet-Membran (fand sich keine Läsion, wurde der Schnitt der Gruppe „beide Seiten intakt“ zugeordnet, bei einer minimalen Läsion bzw. Perforation mindestens einer Seite entsprechend der Gruppe „Minimalläsion“ bzw. „Teilperforation“)

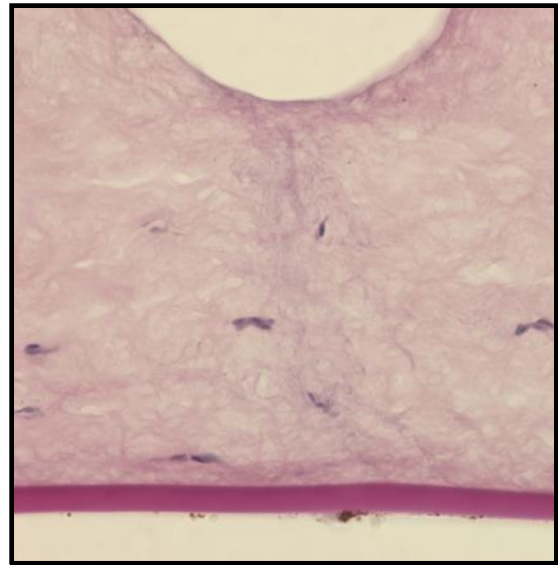

Abb. 3: „beide Seiten intakt" (x 40)

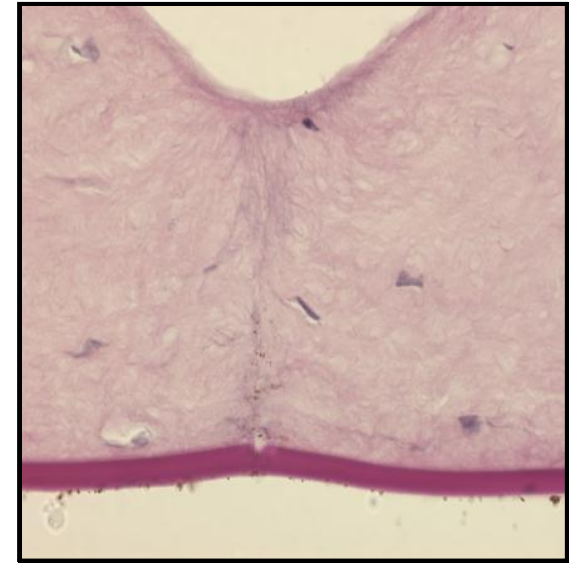

Abb.4: „Minimalläsion““ (x 40) 


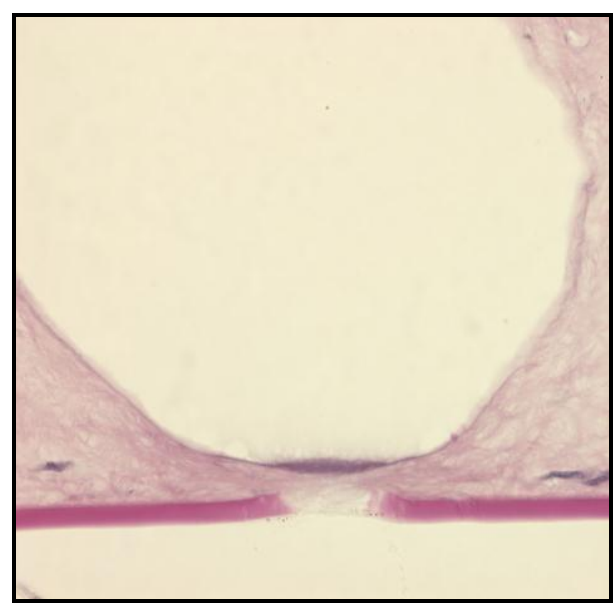

Abb. 5: „Teilperforation“ (x 40)

f. Anzahl vorhandener "Verdichtungszonen“ (= durch thermischen Einfluss oder Kompression entstandene Gewebeverdichtung im Randbereich der Mikroblasen, erkennbar durch ihre tiefviolette Färbung)

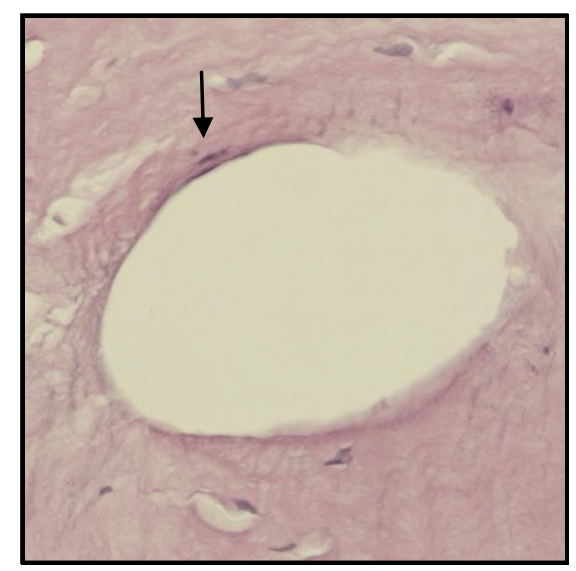

Abb. 6: Verdichtungszone (Pfeil) (x 20)

Für die Auswertung wurden drei verschiedene Gruppen gebildet. Die Gruppeneinteilung lautete dabei wie folgt (vgl. auch Abb. 7):

\section{Gruppe A:}

Intendierter Abstand Bowman-Lamelle zu horizontalem Anteil des Lentikels (sechs Untergruppen):

a. $550 \mu \mathrm{m}$ (neun Schnitte) $\rightarrow$ „Abstand 550“

b. $650 \mu \mathrm{m}$ (zehn Schnitte) $\rightarrow$ „Abstand 650“ 
C. $750 \mu \mathrm{m}$ (neun Schnitte) $\rightarrow$ „Abstand 750“

d. $850 \mu \mathrm{m}$ (zehn Schnitte) $\rightarrow$ „Abstand 850“

e. $950 \mu \mathrm{m}$ (sieben Schnitte) $\rightarrow$ „Abstand 950“

f. $1000 \mu \mathrm{m}$ (neun Schnitte) $\rightarrow$ „Abstand 1000“

\section{Gruppe B:}

Zustand der Descemet-Membran (drei Untergruppen):

a. „beide Seiten intakt" (keine sichtbare Einwirkung des Lasers, 20 Schnitte)

b. „Teilperforation“ (zumindest auf einer Seite vollständige Durchtrennung der Descemet-Membran, elf Schnitte)

c. „Minimalläsion“ (zumindest auf einer Seite eine sichtbare Läsion, 23 Schnitte)

\section{Gruppe C:}

Abstand Descemet-Membran zu horizontalem Anteil des Lentikels $=$ Dicke des Lentikels (drei Untergruppen):

a. 330-630 $\mu \mathrm{m}$ (zwölf Schnitte) $\rightarrow$ „Dicke Lentikel 330“

b. 631-930 $\mu \mathrm{m}$ (29 Schnitte) $\rightarrow$ „Dicke Lentikel 631“

C. 931-1230 $\mu \mathrm{m}$ (13 Schnitte) $\rightarrow$ „Dicke Lentikel 931“

Die Gruppen B und C sind hierbei Einteilungen, die nach der Auswertung getroffen wurden (also nicht vorher festgelegt werden konnten), um etwaige Unterschiede bei einigen Fragestellungen aufzuzeigen. Daraus folgt, dass nur Gruppe A bei allen Fragestellungen untersucht wurde.

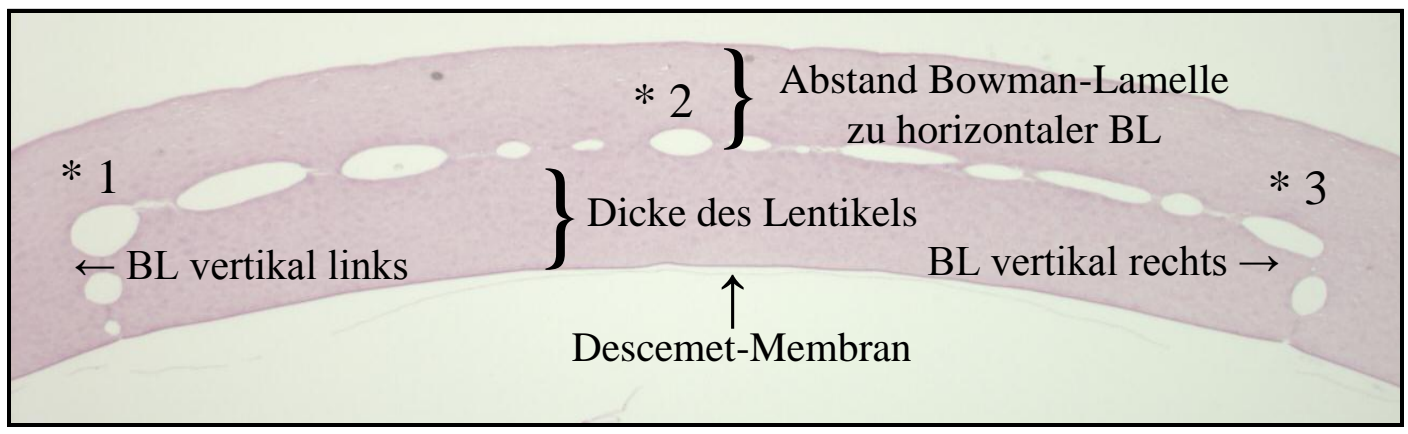

Abb. 7: Gesamtübersicht einer Histologie mit Erläuterungen; $B L=$ Blasenlinie; $* 1=$,links“, *2 = „,mittig“ und *3 = ,rechts“ sind die drei Messpunkte des Abstandes Bowman-Lamelle zur horizontaler Blasenlinie (x 5) 


\subsection{Statistische Methoden}

Die Messergebnisse wurden in eine Datendatei übertragen und mit Hilfe von SPSS für Windows, Version 15.0 (SPSS Inc., Chicago, IL) ausgewertet [3].

Es wurde eine allgemeine deskriptive Statistik erstellt - Minimum (Min), Maximum (Max), Mittelwerte (MW) \pm Standardabweichung (SD), Median (MD) - und für die Feststellung der Unterschiede zwischen bzw. innerhalb der einzelnen Gruppen wurden die Ergebnisse mittels des Wilcoxon-Tests für abhängige Größen, des Mann-Whitney-U-Tests für unabhängige Größen und des Friedman-Tests analysiert. Der Spearman-Korrelationskoeffizient diente der Überprüfung der Rangkorrelationen, Varianzunterschiede wurden mittels des Levene-Tests überprüft. Sämtliche Werte wurden auf die erste Stelle nach dem Komma gerundet. Unterschiede zwischen zwei Gruppen galten als statistisch signifikant, wenn der $p$-Wert kleiner als 0,05 war.

Bei einem insgesamt begrenzten Stichprobenumfang von $n=54$ in der Gesamtstichprobe ergaben sich insbesondere bei den Vergleichen von Teilstichproben Fallzahlen, bei denen eine Prüfung von Verteilungsannahmen, wie sie bei parametrischen Verfahren unterstellt werden, nur bedingt sinnvoll war. Es wurden daher alle Signifikanzprüfungen durchgängig mit parameterfreien Verfahren durchgeführt. 


\section{Ergebnisse}

Die genannten Fragestellungen wurden teilweise in einer Gesamtanalyse aller 54 Histologien bearbeitet, teils aber auch - soweit Unterschiede festgestellt wurden - in den verschiedenen Gruppeneinteilungen.

Fielen innerhalb der Gruppen Unterschiede auf, wurden die Untergruppen miteinander verglichen.

\subsection{Intendierter Abstand der Bowman-Lamelle zur horizontalen Blasenlinie}

Es wurden sechs verschiedene Abstände von der Bowman-Lamelle ausgehend und im Stroma endend am FEMTEC-Laser eingestellt: $550 \mu \mathrm{m}$, $650 \mu \mathrm{m}, 750 \mu \mathrm{m}, 850 \mu \mathrm{m}, 950 \mu \mathrm{m}$ und $1000 \mu \mathrm{m}$.

Diese Abstände zur entstandenen horizontalen Blasenlinie wurden an insgesamt drei Stellen gemessen: in der Mitte des Lentikels („mittig“) sowie an den beiden Enden des horizontalen Blasenverlaufes (,rechts" und „links“, s. auch Skizze auf Seite 19.

Tabelle 1 zeigt den minimalen und maximalen Abstand sowie den errechneten Mittelwert dieser drei Messpunkte ((rechts + mittig + links) / 3) samt Standardabweichung (SD). 


\begin{tabular}{|c|c|c|c|c|c|c|}
\hline$[\mu \mathrm{m}]$ & Min & Max & MW & SD & MD & $\mathbf{n}$ \\
\hline $\begin{array}{c}\text { Abstand } \\
550\end{array}$ & 330 & 505 & 395,9 & 33,1 & 390 & 9 \\
\hline $\begin{array}{c}\text { Abstand } \\
650\end{array}$ & 395 & 660 & 509,8 & 55 & 500,8 & 10 \\
\hline $\begin{array}{c}\text { Abstand } \\
750\end{array}$ & 555 & 700 & 632 & 39,2 & 651,7 & 9 \\
\hline $\begin{array}{c}\text { Abstand } \\
850\end{array}$ & 540 & 860 & 734,5 & 64,8 & 742,5 & 10 \\
\hline $\begin{array}{c}\text { Abstand } \\
950\end{array}$ & 685 & 940 & 790,9 & 77,9 & 765 & 7 \\
\hline $\begin{array}{c}\text { Abstand } \\
1000\end{array}$ & 705 & 1160 & 947,9 & 93,5 & 940 & 9 \\
\hline
\end{tabular}

Tab. 1: Minimum, Maximum, Mittelwert, Standardabweichung \& Median des intendierten Abstandes Bowman-Lamelle zu horizontaler Blasenlinie; Gruppe A (Abstand Bowman-Lamelle zu horizontalem Anteil des Lentikels); $(\mathrm{n}=54)$

Bzgl. des vorher eingestellten Abstandes lässt sich festhalten, dass bei allen Gruppen bis auf die Gruppe „Abstand 1000“ im Mittel eine negative Abweichung zwischen 115-160 $\mu \mathrm{m}$ besteht.

\section{Gleichmäßigkeit des Abstandes Bowman-Lamelle zur horizontalen Blasenlinie}

Wie erwähnt wurden die Abstände zur entstandenen horizontalen Blasenlinie an drei Stellen gemessen. 


\begin{tabular}{|c|c|c|c|c|c|c|c|c|c|c|}
\hline \multirow[t]{2}{*}[\mu\mathrm{m}]{} & \multicolumn{3}{|c|}{ rechts } & \multicolumn{3}{|c|}{ mittig } & \multicolumn{3}{|c|}{ links } & \multirow[t]{2}{*}{$\mathbf{n}$} \\
\hline & MW & SD & MD & MW & SD & MD & MW & SD & MD & \\
\hline $\begin{array}{c}\text { Abstand } \\
550\end{array}$ & 381,7 & 38,7 & 380 & 398,9 & 44,4 & 385 & 407,2 & 52 & 400 & 9 \\
\hline $\begin{array}{c}\text { Abstand } \\
650\end{array}$ & 525,5 & 67,9 & 510 & 501,0 & 50,4 & 505 & 503,0 & 62,3 & 510 & 10 \\
\hline $\begin{array}{c}\text { Abstand } \\
750\end{array}$ & 628,3 & 49,2 & 630 & 632,2 & 47,7 & 635 & 635,6 & 44,3 & 630 & 9 \\
\hline $\begin{array}{c}\text { Abstand } \\
850\end{array}$ & 735,5 & 78,1 & 742,5 & 732,5 & 63,9 & 745 & 735,5 & 77,8 & 760 & 10 \\
\hline $\begin{array}{c}\text { Abstand } \\
950\end{array}$ & 792,9 & 73,9 & 810 & 786,4 & 83,1 & 755 & 793,6 & 91,1 & 805 & 7 \\
\hline $\begin{array}{c}\text { Abstand } \\
1000\end{array}$ & 934,4 & 84,7 & 910 & 922,8 & 111,6 & 920 & 986,7 & 104,2 & 975 & 9 \\
\hline
\end{tabular}

Tab. 2: Mittelwert, Standardabweichung \& Median in $\mu \mathrm{m}$ des intendierten Abstandes Bowman-Lamelle zu horizontaler Blasenlinie unterschieden nach den drei Messpunkten rechts, mittig, links; Gruppe A (Abstand Bowman-Lamelle zu horizontalem Anteil des Lentikels); $(n=54)$

Man sieht, dass an allen drei gewählten Punkten der gemessene Abstand zur Bowman-Lamelle annähernd identisch ist. Die Abweichung zwischen den drei Werten bewegt sich hierbei innerhalb der fünf Untergruppen zwischen 3 und 25,5 $\mu \mathrm{m}$, die höchste Abweichung beträgt gerade einmal 63,9 $\mu \mathrm{m}$ („Abstand 1000“).

Die Unterschiede zwischen den drei Messungen („rechts“, „mittig“, „links“) auf jeder intendierten Abstandsstufe wurden jeweils mittels eines FriedmanTests auf Signifikanz geprüft.

Bei den fünf Abstandsstufen $550(p=0,110), 650(p=0,313), 750$ ( $p=$ $0,971), 850(p=0,614)$ und $950(p=0,764)$ ergeben sich nicht signifikante Unterschiede.

In der mit 1000 höchsten Abstandsstufe ergibt sich im Gegensatz dazu ein signifikanter Unterschied ( $p=0,045$ ). Für diese Abstandsstufe zeigen die Einzelvergleiche zwischen den drei Messungen über Wilcoxon-Tests, dass die Unterschiede zwischen links und rechts $(p=0,044)$ und zwischen links und mittig $(p=0,021)$ jeweils auf dem $5 \%$-Niveau signifikant sind. 


\section{Unterschiede der Standardabweichung in Gruppe A}

Auffallend ist eine steigende Standardabweichung mit zunehmendem Abstand und damit abnehmender Lentikelhöhe (geht man im Schnitt von annähernd gleich dicken Corneae aus) vor allem in der Gruppe „Abstand 1000".

Daher wurden - jeweils getrennt - die drei Messparameter (,rechts“, „mittig“, "links") aller sechs Abstandsstufen mittels des Levene-Tests auf Varianzunterschiede geprüft.

Für die Messungen "rechts“ ist mit $p=0,273$ das Ergebnis deutlich genug nicht signifikant, so dass man von einer Gleichheit der Streuungen ausgehen kann. Ähnliches gilt für die „mittigen“" Messungen, hier mit $p=0,196$. Bei den Messungen „links" ist dagegen zwar mit $p=0,128$ ein Streuungsunterschied nicht nachgewiesen, er kann aber bezogen auf die Grundgesamtheit auch nicht ausgeschlossen werden, da hier die Signifikanz nicht sehr deutlich verfehlt wird.

\section{Unterschiede der Spannweiten in Gruppe A}

Zusätzlich wurden über alle drei Messungen („rechts“, „mittig“, „links“) zusammen genommen die Minimal- und Maximalwerte bestimmt, und zwar jeweils in jeder der sechs Abstandsstufen. Die Differenz dieser beiden Werte für jede Abstandsstufe ist dann die Spannweite der Messungen, die in dieser Abstandsstufe vorkommt.

Berechnet wurde eine Rangkorrelation (Spearman-Koeffizient) zwischen dem intendierten Abstand (Abstandsstufe) einerseits und der Spannweite der Messungen in dieser Stufe. Diese Korrelation wurde einmal für alle sechs Stufen, also mit einer Fallzahl von sechs, berechnet und einmal nur für die unteren fünf Stufen unter Auslassung der höchsten Stufe (= „Abstand 1000“). Für alle sechs Stufen ergibt sich eine recht hohe Korrelation von $r=0,600$, die jedoch bei nur $n=6$ Fällen mit $p=0,208$ nicht signifikant ist.

Allerdings kann man bei dieser kleinen Fallzahl zumindest einen Trend in der Stichprobe erkennen, dass tendenziell die Spannweiten steigen, wenn der intendierte Abstand größer wird. 
4.2 Anzahl der Blasen

\begin{tabular}{|c|c|c|c|c|c|c|}
\hline$[\mathrm{n}]$ & Min & Max & MW & SD & MD & n \\
\hline $\begin{array}{c}\text { vertikal } \\
\text { rechts }\end{array}$ & 0 & 9 & 2,7 & 1,6 & 3,0 & 54 \\
\hline $\begin{array}{c}\text { vertikal } \\
\text { links }\end{array}$ & 0 & 11 & 2,7 & 1,8 & 3,0 & 54 \\
\hline \begin{tabular}{c} 
horizontal \\
\hline
\end{tabular} & 9 & 28 & 15,5 & 3,5 & 15,0 & 54 \\
\hline
\end{tabular}

Tab. 3: Minimum, Maximum, Mittelwert, Standardabweichung \& Median als absoluter Wert der Blasenanzahl im Bereich der Exzision; Gesamtstatistik; $(\mathrm{n}=54)$

\begin{tabular}{|c|c|c|c|c|c|c|c|c|c|c|}
\hline & MW & SD & MD & MW & SD & MD & MW & SD & MD & \\
\cline { 2 - 13 } & \multicolumn{3}{|c|}{ rechts } & \multicolumn{3}{|c|}{ links } & \multicolumn{2}{|c|}{ horizontal } & n \\
\hline $\begin{array}{c}\text { Abstand } \\
550\end{array}$ & 4,3 & 1,5 & 4,0 & 4,2 & 2,9 & 3,0 & 13,2 & 2,3 & 13,0 & 9 \\
\hline $\begin{array}{c}\text { Abstand } \\
650\end{array}$ & 3,5 & 2,0 & 3,0 & 3,5 & 1,1 & 3,5 & 17,3 & 2,9 & 17,5 & 10 \\
\hline $\begin{array}{c}\text { Abstand } \\
750\end{array}$ & 2,8 & 1,4 & 3,0 & 3,1 & 1,2 & 3,0 & 14,8 & 2,4 & 14,0 & 9 \\
\hline $\begin{array}{c}\text { Abstand } \\
850\end{array}$ & 2,4 & 0,8 & 3,0 & 2,5 & 1,3 & 2,0 & 14,8 & 2,6 & 14,5 & 10 \\
\hline $\begin{array}{c}\text { Abstand } \\
950\end{array}$ & 0,4 & 0,9 & 1,0 & 1,6 & 0,9 & 2,0 & 15,1 & 3,2 & 15,0 & 7 \\
\hline $\begin{array}{c}\text { Abstand } \\
1000\end{array}$ & 1,6 & 0,9 & 2,0 & 1,1 & 0,8 & 1,0 & 17,7 & 5,4 & 15,0 & 9 \\
\hline
\end{tabular}

Tab. 4: Mittelwert, Standardabweichung \& Median als absoluter Wert der Blasenanzahl im Bereich der Exzision unterschieden nach den drei Seiten vertikal rechts, vertikal links und horizontal; Gruppe A (Abstand Bowman-Lamelle zu horizontalem Anteil des Lentikels); $(n=54)$

Die Anzahl der Blasen in den beiden vertikalen Ebenen verringert sich erwartungsgemäß mit zunehmendem Abstand Bowman-Lamelle zu Lentikel 
und damit mit der niedrigeren Höhe des Lentikels. Dies gilt, wenn man von annähernd gleich dicken Corneae ausgeht. Die Anzahl in der horizontalen Ebene bleibt auch in den Untergruppen nahezu gleich $(p=0,266)$.

Mittels Rangkorrelationen mit dem Spearman-Korrelationskoeffizienten wurde nun überprüft, ob der vermutete Zusammenhang, dass mit zunehmendem Abstand die Blasenanzahl tatsächlich geringer ist, zutrifft.

Die Ergebnisse stützen die aufgrund der deskriptiven Statistik getroffenen Annahmen. Die Beziehungen zwischen intendiertem Abstand und Blasenanzahl in den beiden vertikalen Ebenen weisen eine starke negative Korrelation in Höhe von $r=-0,626$ (links) und $r=-0,706$ (rechts) auf. Beide Korrelationen sind bei $\mathrm{n}=54$ als Stichprobenumfang höchst signifikant $(p<0,001)$. Die Abnahme der Blasenanzahl mit zunehmendem Abstand Bowman-Lamelle zu Lentikel ist damit in der vertikalen Ebene nachgewiesen. Mit dem gleichen Verfahren wurde die Blasenanzahl der horizontalen Linie überprüft. Die Korrelation in dieser Ebene ist in der Stichprobe mit $r=0,154$ nur sehr schwach ausgeprägt und mit $p=0,266$ auch so deutlich nicht signifikant, dass man unterstellen kann, dass hier wirklich auch in der Grundgesamtheit kein Zusammenhang besteht, d.h., dass es keine Unterschiede in der Blasenanzahl in der horizontalen Ebene in den Untergruppen gibt.

Auf den beiden vertikalen Seiten sind die jeweiligen Mittelwerte, also die Blasenanzahl, innerhalb der Untergruppen nahezu identisch. Es wurde daher vermutet, dass der Laser auf beiden Seiten gleich arbeitet. 
Die Unterschiede zwischen den beiden vertikalen Seiten wurden für jede Stufe des intendierten Abstandes getrennt mit Wilcoxon-Tests untersucht.

\begin{tabular}{|c|c|c|}
\hline $\begin{array}{c}\text { Abstand } \\
550\end{array}$ & 0,76 & $\mathbf{n}$ \\
\hline $\begin{array}{c}\text { Abstand } \\
650\end{array}$ & 0,73 & 10 \\
\hline $\begin{array}{c}\text { Abstand } \\
750\end{array}$ & 0,52 & 9 \\
\hline $\begin{array}{c}\text { Abstand } \\
850\end{array}$ & 0,90 & 10 \\
\hline $\begin{array}{c}\text { Abstand } \\
950\end{array}$ & 0,66 & 7 \\
\hline $\begin{array}{c}\text { Abstand } \\
1000\end{array}$ & 0,38 & 9 \\
\hline
\end{tabular}

Tab. 5: Signifikanzniveau p für den Vergleich der Blasenanzahl im Bereich der Exzision vertikal links mit vertikal rechts; Gruppe A (Abstand Bowman-Lamelle zu horizontalem Anteil des Lentikels); $(n=54)$

Damit ist nachgewiesen, dass der FEMTEC-Laser auf beiden vertikalen Schnitten die gleiche Anzahl an Blasen in den jeweiligen Abstandsstufen produziert. 


\subsection{Länge und Breite der Blasen}

Vergleich der Länge und Breite der Blasen horizontal vs. vertikal

Die Länge und Breite der Blasen wurde wie folgt gemessen:

\begin{tabular}{|c|c|c|c|c|c|c|c|c|c|c|}
\hline$[\mu \mathrm{m}]$ & Min & SD & MD & $\operatorname{Max}$ & SD & MD & MW & SD & MD & $\mathbf{n}$ \\
\hline $\begin{array}{l}\text { vertikal } \\
\text { rechts }\end{array}$ & 20,0 & 73,5 & 102,5 & 590,0 & 101,9 & 225,0 & 158,4 & 70,6 & 146,7 & 54 \\
\hline $\begin{array}{c}\text { vertikal } \\
\text { links }\end{array}$ & 15,0 & 73,2 & 87,5 & 595,0 & 115,9 & 200,0 & 160,9 & 75,7 & 149,0 & 54 \\
\hline horizontal & 15,0 & 34,6 & 50,0 & 1600,0 & 228,9 & 560,0 & 250,5 & 68,7 & 243,9 & 54 \\
\hline
\end{tabular}

Tab. 6: Minimum, Maximum, Mittelwert, jeweils mit Standardabweichung \& Median der Länge der Blasen im Bereich der Exzision unterschieden nach den drei Seiten vertikal rechts, vertikal links und horizontal; Gesamtstatistik; $(n=54)$

\begin{tabular}{|c|c|c|c|c|c|c|c|c|c|c|c|}
\hline [ $\mu \mathrm{m}]$ & Min & SD & MD & \multicolumn{1}{|c|}{ Max } & \multicolumn{2}{c|}{ SD } & MD & MW & SD & MD & n \\
\hline $\begin{array}{c}\text { vertikal } \\
\text { rechts }\end{array}$ & 15,5 & 53,7 & 62,5 & 385,0 & 66,0 & 162,5 & 117,4 & 50,1 & 111,0 & 54 \\
\hline $\begin{array}{c}\text { vertikal } \\
\text { links }\end{array}$ & 10,0 & 51,8 & 62,5 & 360,0 & 69,6 & 162,5 & 119,3 & 48,4 & 112,0 & 54 \\
\hline horizontal & 10,0 & 22,5 & 30,0 & 425,0 & 48,6 & 230,0 & 132,9 & 26,3 & 129,5 & 54 \\
\hline
\end{tabular}

Tab. 7: Minimum, Maximum, Mittelwert, jeweils mit Standardabweichung \& Median der Breite der Blasen im Bereich der Exzision unterschieden nach den drei Seiten vertikal rechts, vertikal links und horizontal; Gesamtstatistik; $(n=54)$

Die durchschnittliche Blasenlänge und -breite in der Horizontalen ist höher als in der Vertikalen; die Differenz der Mittelwerte bzgl. der Länge beträgt 89,6 $\mu \mathrm{m}$ (rechts) bzw. 92,1 $\mu \mathrm{m}$ (links) (vgl. Tab. 6). 
Bzgl. der Breite der Blasen ist dieser Unterschied der horizontalen mit der vertikalen Seite im Vergleich zur rechten Seite signifikant $(p=0,034)$, im Vergleich zur linken wird die Signifikanz nur knapp verfehlt $(p=0,062$, Wilcoxon-Test).

Der Unterschied der Länge ist im Vergleich der Horizontalen zu beiden vertikalen Seiten höchst signifikant (jeweils $p<0,001$ ). Es kann damit gezeigt werden, dass die Blasen in der Horizontalen länger und zumindest im Vergleich zur rechten Seite auch breiter sind als in der Vertikalen.

Beim Vergleich der beiden vertikalen Seiten miteinander fällt auf, dass es keine Unterschiede in Länge und Breite der Blasen zu geben scheint.

Für die Länge $(p=0,913)$ und die Breite $(p=0,831)$ der Blasen ergibt sich hier kein signifikanter Unterschied zwischen links und rechts (Wilcoxon-Test).

\section{Vergleich der Länge und Breite der Blasen für Gruppe A}

\begin{tabular}{|c|c|c|c|c|c|c|c|c|c|c|c|}
\hline \multirow{2}{*}[\mu\mathrm{m}]{} & \multicolumn{2}{|c|}{ vertikal rechts } & \multicolumn{2}{|c|}{ vertikal links } & \multicolumn{3}{|c|}{ horizontal } & n \\
\cline { 2 - 10 } & MW & SD & MD & MW & SD & MD & MW & SD & MD & \\
\hline $\begin{array}{c}\text { Abstand } \\
550\end{array}$ & 134,3 & 54,0 & 138,3 & 153,7 & 105,6 & 148,0 & 317,9 & 79,2 & 302,3 & 9 \\
\hline $\begin{array}{c}\text { Abstand } \\
650\end{array}$ & 140,4 & 30,1 & 132,9 & 160,9 & 71,7 & 137,2 & 232,6 & 48,1 & 228,2 & 10 \\
\hline $\begin{array}{c}\text { Abstand } \\
750\end{array}$ & 215,2 & 97,7 & 250,0 & 182,5 & 74,7 & 175,0 & 236,6 & 46,7 & 227,2 & 9 \\
\hline $\begin{array}{c}\text { Abstand } \\
850\end{array}$ & 120,2 & 62,5 & 127,5 & 157,8 & 53,2 & 157,5 & 281,9 & 65,9 & 274,4 & 10 \\
\hline $\begin{array}{c}\text { Abstand } \\
950\end{array}$ & 203,5 & 70,1 & 200,0 & 148,2 & 60,3 & 143,8 & 205,4 & 37,9 & 217,7 & 7 \\
\hline $\begin{array}{c}\text { Abstand } \\
1000\end{array}$ & 157,7 & 56,3 & 171,3 & 158,1 & 94,9 & 132,5 & 217,1 & 65,0 & 233,9 & 9 \\
\hline
\end{tabular}

Tab. 8: Mittelwert, Standardabweichung \& Median der Länge der Blasen im Bereich der Exzision unterschieden nach den drei Seiten vertikal rechts, vertikal links und horizontal; Gruppe A (Abstand Bowman-Lamelle zu horizontalem Anteil des Lentikels); $(\mathrm{n}=54)$ 


\begin{tabular}{|c|c|c|c|c|c|c|c|c|c|c|}
\hline \multirow[t]{2}{*}[\mu\mathrm{m}]{} & \multicolumn{3}{|c|}{ vertikal rechts } & \multicolumn{3}{|c|}{ vertikal links } & \multicolumn{3}{|c|}{ horizontal } & \multirow[t]{2}{*}{$\mathbf{n}$} \\
\hline & MW & SD & MD & MW & SD & MD & MW & SD & MD & \\
\hline $\begin{array}{c}\text { Abstand } \\
550\end{array}$ & 106,1 & 38,7 & 113,8 & 120,1 & 57,4 & 126,7 & 144,4 & 35,7 & 139,4 & 9 \\
\hline $\begin{array}{c}\text { Abstand } \\
650\end{array}$ & 119,2 & 35,3 & 123,3 & 128,4 & 46,6 & 117,1 & 135,6 & 17,9 & 127,8 & 10 \\
\hline $\begin{array}{c}\text { Abstand } \\
750\end{array}$ & 149,6 & 74,7 & 165,0 & 131,5 & 45,0 & 121,7 & 130,8 & 19,4 & 128,6 & 9 \\
\hline $\begin{array}{c}\text { Abstand } \\
850\end{array}$ & 88,2 & 45,7 & 88,3 & 121,2 & 44,9 & 127,5 & 143,5 & 24,8 & 135,7 & 10 \\
\hline $\begin{array}{c}\text { Abstand } \\
950\end{array}$ & 140,8 & 45,3 & 150,0 & 102,5 & 38,6 & 97,5 & 126,9 & 27,5 & 135,3 & 7 \\
\hline $\begin{array}{c}\text { Abstand } \\
1000\end{array}$ & 110,9 & 34,4 & 98,7 & 103,8 & 61,1 & 85,0 & 113,5 & 23,8 & 118,4 & 9 \\
\hline
\end{tabular}

Tab. 9: Mittelwert, Standardabweichung \& Median der Breite der Blasen im Bereich der Exzision unterschieden nach den drei Seiten vertikal rechts, vertikal links und horizontal; Gruppe A (Abstand Bowman-Lamelle zu horizontalem Anteil des Lentikels); $(\mathrm{n}=54)$

Innerhalb der Gruppen wurden die beiden gleichen Fragestellungen wie oben untersucht (s. 4.3).

Dabei zeigt sich, dass in den Gruppen „Abstand 550“ (links p =0,008; rechts $p=0,008$ ), „Abstand 650“ (links $p=0,017$; rechts $p=0,007$ ) und „Abstand 850“ (links $p=0,009$; rechts $p=0,007$ ) die längeren Blasen in der Horizontalen statistisch nachgewiesen werden konnten; sowohl im Vergleich zur linken als auch zur rechten Seite. Für die anderen Gruppen trifft dies nicht zu.

In keiner der Gruppen sind die Blasen in der Horizontalen breiter als in der Vertikalen.

Analog zu oben wurden nun noch einmal die beiden vertikalen Seiten separat in den sechs Abstandsstufen miteinander verglichen.

Bezüglich der Länge der Blasen zeigen sich in vier der sechs Fälle keine Unterschiede (550: $p=0,767 ;$ 650: $p=0,333 ;$ 750: $p=0,441$; 1000: $p=0,735)$. 
Bei „Abstand 950“ sind die Unterschiede mit $p=0,043$ trotz kleinerer Fallzahl $(n=5)$ knapp signifikant. Die Länge der Blasen ist nachweislich links größer als rechts. Bei „Abstand 850“ ist bei zehn Fällen mit $p=0,086$ statistisch keine klare Aussage möglich.

Ähnlich sieht es bei der Breite der Blasen aus. Hier gibt es keine signifikanten Unterschiede bei denselben vier Abstandsstufen, in denen schon bzgl. der Länge kein Unterschied gefunden wurde (550: $p=0,484$; 650: $p=0,508 ; 750: p=0,767 ; 1000: p=0,612$ ). Bei den Abstandsstufen 850 und 950 wird dagegen mit $p=0,074$ bzw. $p=0,080$ die Signifikanz nur sehr knapp verfehlt, was in Anbetracht der Stichprobengröße von zehn bzw. fünf andeutet, dass hier in der Grundgesamtheit doch Unterschiede zwischen den Seiten bestehen könnten.

\subsection{Verhältnis Blasenlänge zur Länge der dazwischen liegenden Gewebestege}

\section{Das Verhältnis in der Gesamtbetrachtung}

Es wurde berechnet, wie sich die Länge der Blasen zur gemessenen Länge der zwischen den Blasen liegenden Gewebestege verhält. Im weiteren Verlauf wird gezeigt, ob dieses Verhältnis einen Einfluss auf den Gewebezustand dieser Stege hat.

\begin{tabular}{|c|c|c|c|}
\hline & Verhältnis & SD & n \\
\hline $\begin{array}{c}\text { vertikal } \\
\text { rechts }\end{array}$ & 2,0 & 1,4 & 54 \\
\hline $\begin{array}{c}\text { vertikal } \\
\text { links }\end{array}$ & 1,8 & 1,2 & 54 \\
\hline horizontal & 3,6 & 1,7 & 54 \\
\hline
\end{tabular}

Tab. 10: Mittelwert \& Standardabweichung als absoluter Wert des Verhältnisses Blasenlänge zur Länge der dazwischen liegenden Gewebestege; Gesamtstatistik; $(\mathrm{n}=54)$ 
In der horizontalen Ebene ist die Blasenlänge im Verhältnis zur Länge der dazwischen liegenden Gewebestege anteilig deutlich höher als in der vertikalen Ebene.

Mittels des Wilcoxon-Tests wurde diese Aussage überprüft. Es zeigt sich ein Signifikanzniveau für den Vergleich beider Seiten mit der horizontalen Seite von jeweils $p<0,001$.

Für den Vergleich der beiden vertikalen Seiten untereinander zeigt sich auf der Ebene aller Proben über alle Abstandsstufen hinweg deskriptiv eine gewisse Tendenz, dass dieses Verhältnis in der Vertikalen rechts höher ist als links, dennoch wird die Signifikanz mit $p=0,102$ bei $n=50$ hier verfehlt.

\section{Das Verhältnis in Gruppe A}

Das Verhältnis in Gruppe A ist wie folgt:

\begin{tabular}{|c|c|c|c|c|c|c|c|}
\hline & \multicolumn{2}{|c|}{ vertikal rechts } & \multicolumn{2}{|c|}{ vertikal links } & \multicolumn{2}{|c|}{ horizontal } & n \\
\cline { 2 - 9 } & MW & SD & MW & SD & MW & SD & \\
\hline $\begin{array}{c}\text { Abstand } \\
550\end{array}$ & 1,9 & 1,3 & 1,5 & 0,9 & 4,2 & 2,1 & 9 \\
\hline $\begin{array}{c}\text { Abstand } \\
650\end{array}$ & 1,6 & 0,8 & 1,9 & 0,9 & 3,5 & 1,5 & 10 \\
\hline $\begin{array}{c}\text { Abstand } \\
750\end{array}$ & 2,7 & 1,7 & 2,0 & 1,0 & 2,8 & 0,9 & 9 \\
\hline $\begin{array}{c}\text { Abstand } \\
850\end{array}$ & 1,8 & 1,5 & 2,2 & 1,8 & 5,0 & 2,0 & 10 \\
\hline $\begin{array}{c}\text { Abstand } \\
950\end{array}$ & 1,9 & 0,8 & 1,3 & 0,8 & 3,1 & 1,7 & 7 \\
\hline $\begin{array}{c}\text { Abstand } \\
1000\end{array}$ & 2,3 & 1,8 & 1,5 & 1,1 & 2,6 & 0,7 & 9 \\
\hline
\end{tabular}

Tab. 11: Mittelwert \& Standardabweichung als absoluter Wert des Verhältnisses Blasenlänge zur Länge der dazwischen liegenden Gewebestege; Gruppe A (Abstand Bowman-Lamelle zu horizontalem Anteil des Lentikels); $(\mathrm{n}=54)$ 
Entsprechend zu oben die Signifikanzen für Gruppe A:

\begin{tabular}{|c|c|c|c|}
\hline $\begin{array}{c}\text { Abstand } \\
550\end{array}$ & $\mathrm{p}=0,011$ & $\mathrm{p}=0,008$ & $\mathbf{n}$ \\
\hline $\begin{array}{c}\text { Abstand } \\
650\end{array}$ & $\mathrm{p}=0,005$ & $\mathrm{p}=0,022$ & 10 \\
\hline $\begin{array}{c}\text { Abstand } \\
750\end{array}$ & $\mathrm{p}=0,859$ & $\mathrm{p}=0,066$ & 9 \\
\hline $\begin{array}{c}\text { Abstand } \\
850\end{array}$ & $\mathrm{p}=0,007$ & $\mathrm{p}=0,009$ & 10 \\
\hline $\begin{array}{c}\text { Abstand } \\
950\end{array}$ & $\mathrm{p}=0,116$ & $\mathrm{p}=0,046$ & 7 \\
\hline $\begin{array}{c}\text { Abstand } \\
1000\end{array}$ & $\mathrm{p}=0,575$ & $\mathrm{p}=0,036$ & 9 \\
\hline
\end{tabular}

Tab. 12: Signifikanzniveau $\mathbf{p}$ für den Vergleich des Verhältnisses Blasenlänge zur Länge der dazwischen liegenden Gewebestege (jeweils horizontal mit vertikal rechts bzw. links); Gruppe A (Abstand Bowman-Lamelle zu horizontalem Anteil des Lentikels); $(\mathrm{n}=54)$

Man stellt fest, dass p für die Gruppen „Abstand 950“ und „Abstand 1000“ zumindest für die rechte Seite nicht signifikant ist. In Gruppe „Abstand 750“ trifft dies für beide Seiten zu.

Damit bestätigt sich größtenteils auch innerhalb der Gruppeneinteilung, dass die Blasen in der Horizontalen einen höheren Anteil an der Schnittlinie haben.

Analog wurden die beiden vertikalen Seiten differenziert nach intendierten Abständen - bei dann entsprechend verringerter Fallzahl - verglichen. Es zeigt sich in allen sechs Fällen ein nicht signifikantes Ergebnis ( $p=0,441$ für Abstand 550, $p=0,575$ für Abstand 650, $p=0,214$ für Abstand 750, $p=$ 0,959 für Abstand 850, $p=0,138$ für Abstand 950 und $p=0,310$ für Abstand 1000 bei einem $n$ zwischen sieben und zehn).

Lediglich bei „Abstand 950“ ist das Ergebnis zwar auch nicht signifikant, allerdings wird die Signifikanz relativ knapp verfehlt, so dass statistisch keine klare Aussage möglich ist. 
Die Bewertung der Gruppe A weist also in die Richtung, dass das Verhältnis auf beiden vertikalen Seiten gleich ist.

\subsection{Zustand der Gewebestege zwischen den Blasen}

\section{Zusammenhang zwischen Verhältnis „Blasenlänge zu Gewebestege“ und Gewebezustand in der Gesamtbetrachtung}

Die Gewebestege zwischen den Blasen wurden nach der Beschaffenheit der Stromalamellen beurteilt. Der überwiegende Teil zeigt keine direkte oder indirekte Lasereinwirkung.

Für die übrigen Fälle, in denen eine Einwirkung auf das Stroma zu sehen war, wurden die zwei Einteilungen "vakuolig" und „aufgelockert" (vgl. Abb. 1 und 2) getroffen und ausgewertet, wie oft Gewebestege mit diesen Gewebebeschaffenheiten vorkamen.

Zunächst wurden alle 54 Schnitte beurteilt. Es zeigt sich, dass in beiden vertikalen Schnittebenen die Anzahl von „vakuoligen“ bzw. „aufgelockerten“ Gewebestegen im Durchschnitt $<1,0$ ist, hingegen finden sich in der horizontalen Ebene 2,6 \pm 1,7 „aufgelockerte“ bzw. 4,7 \pm 3,3 „vakuolige“ Gewebestege. Der Unterschied ist mit $p<0,001$ höchst signifikant.

Mittels des Mann-Whitney-U-Tests wurde für beide Gewebebeschaffenheiten berechnet, ob ein Zusammenhang zwischen dem Verhältnis Blasenlänge zu Gewebestege und der Gewebebeschaffenheit statistisch nachweisbar ist.

In allen Ebenen wurde jedoch nur ein $p>0,05$ ermittelt, so dass sich kein signifikanter Einfluss nachweisen ließ. 
Zusammenhang zwischen Verhältnis „Blasenlänge zu Gewebestege“ und Gewebezustand in Gruppe A

\begin{tabular}{|c|c|c|c|c|c|c|c|c|c|c|}
\hline & \multicolumn{2}{|c|}{ vertikal rechts } & \multicolumn{3}{c|}{ vertikal links } & \multicolumn{3}{|c|}{ horizontal } & n \\
\hline & MW & SD & MD & MW & SD & MD & MW & SD & MD & \\
\hline $\begin{array}{c}\text { Abstand } \\
550\end{array}$ & 0,0 & - & - & 0,1 & 0,3 & 0,0 & 5,1 & 3,8 & 7,0 & 9 \\
\hline $\begin{array}{c}\text { Abstand } \\
650\end{array}$ & 0,4 & 0,9 & 0,0 & 0,2 & 0,4 & 0,0 & 5,0 & 3,3 & 4,5 & 10 \\
\hline $\begin{array}{c}\text { Abstand } \\
750\end{array}$ & 0,0 & - & - & 0,4 & 0,7 & 0,0 & 3,3 & 3,7 & 3,0 & 9 \\
\hline $\begin{array}{c}\text { Abstand } \\
850\end{array}$ & 0,0 & - & - & 0,0 & - & - & 4,6 & 3,9 & 4,5 & 10 \\
\hline $\begin{array}{c}\text { Abstand } \\
950\end{array}$ & 0,0 & - & - & 0,1 & 0,4 & 0,0 & 4,9 & 3,3 & 4,0 & 7 \\
\hline $\begin{array}{c}\text { Abstand } \\
1000\end{array}$ & 0,0 & - & - & 0,0 & - & - & 5,4 & 1,7 & 6,0 & 9 \\
\hline
\end{tabular}

Tab. 13: Mittelwert, Standardabweichung \& Median der absoluten Anzahl der vakuoligen Gewebestege unterschieden nach den drei Seiten vertikal rechts, vertikal links und horizontal; Gruppe A (Abstand Bowman-Lamelle zu horizontalem Anteil des Lentikels); $(\mathrm{n}=54)$ 


\begin{tabular}{|c|c|c|c|c|c|c|c|c|c|c|}
\hline & \multicolumn{3}{|c|}{ vertikal rechts } & \multicolumn{3}{|c|}{ vertikal links } & \multicolumn{3}{|c|}{ horizontal } & \multirow[t]{2}{*}{$\mathbf{n}$} \\
\hline & MW & SD & MD & MW & SD & MD & MW & SD & MD & \\
\hline $\begin{array}{c}\text { Abstand } \\
550\end{array}$ & 0,1 & 0,3 & 0,0 & 0,3 & 0,7 & 0,0 & 1,9 & 1,2 & 2,0 & 9 \\
\hline $\begin{array}{c}\text { Abstand } \\
650\end{array}$ & 0,1 & 0,3 & 0,0 & 0,6 & 1,6 & 0,0 & 2,8 & 1,7 & 3,0 & 10 \\
\hline $\begin{array}{l}\text { Abstand } \\
750\end{array}$ & 0,1 & 0,3 & 0,0 & 0,1 & 0,3 & 0,0 & 2,3 & 2,1 & 2,0 & 9 \\
\hline $\begin{array}{c}\text { Abstand } \\
850\end{array}$ & 0,1 & 0,3 & 0,0 & 0,0 & - & - & 1,7 & 1,5 & 1,0 & 10 \\
\hline $\begin{array}{c}\text { Abstand } \\
950\end{array}$ & 0,1 & 0,4 & 0,0 & 0,1 & 0,4 & 0,0 & 2,7 & 1,4 & 3,0 & 7 \\
\hline $\begin{array}{c}\text { Abstand } \\
1000\end{array}$ & 0,0 & - & - & 0,2 & 0,4 & 0,0 & 4,2 & 0,8 & 4,0 & 9 \\
\hline
\end{tabular}

Tab. 14: Mittelwert, Standardabweichung \& Median der absoluten Anzahl der aufgelockerten Gewebestege unterschieden nach den drei Seiten vertikal rechts, vertikal links und horizontal; Gruppe A (Abstand Bowman-Lamelle zu horizontalem Anteil des Lentikels); $(\mathrm{n}=54)$

Auch hier findet man - statistisch gesehen - jeweils weniger als einen „vakuoligen“ oder „aufgelockerten“ Gewebesteg auf beiden vertikalen Seiten. Wie in der Gesamtstatistik kommen deskriptiv die „vakuoligen“ auch in den Untergruppen häufiger vor als die „aufgelockerten“ Gewebestege.

Dies ist jedoch statistisch - bis auf die Gruppe Abstand 850 - nicht signifikant, wie die folgende Tabelle zeigt: 


\begin{tabular}{|c|c|c|}
\hline $\begin{array}{c}\text { Abstand } \\
550\end{array}$ & $\begin{array}{c}\text { vakuolig vs. } \\
\text { aufgelockert }\end{array}$ & n \\
\hline $\begin{array}{c}\text { Abstand } \\
650\end{array}$ & $\mathrm{p}=0,056$ & 9 \\
\hline $\begin{array}{c}\text { Abstand } \\
750\end{array}$ & $\mathrm{p}=0,5050$ & 10 \\
\hline $\begin{array}{c}\text { Abstand } \\
850\end{array}$ & $\mathrm{p}=0,044$ & 10 \\
\hline $\begin{array}{c}\text { Abstand } \\
950\end{array}$ & $\mathrm{p}=0,212$ & 7 \\
\hline $\begin{array}{c}\text { Abstand } \\
1000\end{array}$ & $\mathrm{p}=0,084$ & 9 \\
\hline
\end{tabular}

Tab. 15: Signifikanzniveau p für den Vergleich der Anzahl der „vakuoligen“ mit den ,aufgelockerten“ Gewebestegen im horizontalen Bereich der Exzision; Gruppe A (Abstand Bowman-Lamelle zu horizontalem Anteil des Lentikels); $(\mathrm{n}=54)$

Analog zu oben wurde mittels des Mann-Whitney-U-Tests berechnet, ob ein Einfluss des Verhältnisses auf die Gewebebeschaffenheit vorhanden ist. Nur in einer Gruppe zeigt sich ein signifikanter Einfluss des Verhältnisses auf den Zustand des Gewebes, und zwar in der Horizontalen der Gruppe „Abstand 750“ bzgl. der Gewebsbeschaffenheit „vakuolig“ mit einer Signifikanz von $p=0,048$.

\subsection{Anzahl der Verdichtungszonen}

Es wurde jeweils geprüft, ob im Randbereich der Blasen eine Verdichtungszone vorhanden ist.

In $88,9 \%$ der Fälle findet sich mindestens eine Verdichtungszone, von diesen weisen 57,4\% eine zweite auf. Die Breite der Verdichtungszonen bewegt sich hierbei zwischen 1,25 und 2,5 $\mu \mathrm{m}$. Weitere Verdichtungszonen konnten nicht nachgewiesen werden.

In Gruppe A ist die Verteilung wie folgt: 


\begin{tabular}{|c|c|c|c|}
\hline$\%$ & $\begin{array}{c}\text { mindestens eine } \\
\text { Verdichtungszone }\end{array}$ & $\begin{array}{c}\text { davon > eine } \\
\text { Verdichtungszone }\end{array}$ & $\mathbf{n}$ \\
\hline $\begin{array}{c}\text { Abstand } \\
550\end{array}$ & 77,8 & 33,3 & 9 \\
\hline $\begin{array}{c}\text { Abstand } \\
650\end{array}$ & 100 & 90,0 & 10 \\
\hline $\begin{array}{c}\text { Abstand } \\
750\end{array}$ & 88,9 & 44,4 & 9 \\
\hline $\begin{array}{c}\text { Abstand } \\
850\end{array}$ & 90,0 & 70,0 & 10 \\
\hline $\begin{array}{c}\text { Abstand } \\
950\end{array}$ & 85,7 & 57,1 & 7 \\
\hline $\begin{array}{c}\text { Abstand } \\
1000\end{array}$ & 88,9 & 44,4 & 9 \\
\hline
\end{tabular}

Tab. 16: Häufigkeit der Verdichtungszonen; Gruppe A (Abstand BowmanLamelle zu horizontalem Anteil des Lentikels); $(\mathrm{n}=54)$

\subsection{Zustand der Descemet-Membran}

\section{Zustand der Descemet-Membran in der Gesamtbetrachtung}

Der Zustand der Descemet-Membran wurde beurteilt und die Schnitte im Anschluss der entsprechenden Kategorie zugeordnet.

Die Verteilung ist wie folgt:

\begin{tabular}{|c|c|c|}
\hline n & $\begin{array}{c}\text { relative } \\
\text { Häufigkeit in \% }\end{array}$ \\
\hline beide Seiten intakt & 20 & 37,0 \\
\hline Teilperforation & 11 & 20,4 \\
\hline Minimalläsion & 23 & 42,6 \\
\hline
\end{tabular}

Tab. 17: Zustand der Descemet-Membran, Anzahl und relative Häufigkeit in \%; Gesamtstatistik; $(\mathrm{n}=54)$ 
Nur in vier Schnitten (= 7,4 \%) findet sich eine beidseitige Perforation der Descemet-Membran.

\section{Zustand der Descemet-Membran in Gruppe A}

In Gruppe A ist die Verteilung wie folgt:

\begin{tabular}{|c|c|c|c|c|c|c|c|}
\hline & \multicolumn{2}{|c|}{$\begin{array}{c}\text { beide Seiten } \\
\text { intakt }\end{array}$} & \multicolumn{2}{|c|}{ Teilperforation } & \multicolumn{2}{|c|}{ Minimalläsion } & \multirow[t]{2}{*}{$\mathbf{n}$} \\
\hline & $\mathbf{n}$ & $\%$ & $\mathbf{n}$ & $\%$ & $\mathbf{n}$ & $\%$ & \\
\hline $\begin{array}{c}\text { Abstand } \\
550\end{array}$ & 3 & 33,3 & 0 & 0,0 & 6 & 66,6 & 9 \\
\hline $\begin{array}{c}\text { Abstand } \\
650\end{array}$ & 3 & 30,0 & 5 & 50,0 & 2 & 20,0 & 10 \\
\hline $\begin{array}{l}\text { Abstand } \\
750\end{array}$ & 0 & 0,0 & 3 & 33,3 & 6 & 66,6 & 9 \\
\hline $\begin{array}{c}\text { Abstand } \\
850\end{array}$ & 2 & 20,0 & 2 & 20,0 & 6 & 60,0 & 10 \\
\hline $\begin{array}{l}\text { Abstand } \\
950\end{array}$ & 5 & 71,4 & 0 & 0,0 & 2 & 28,6 & 7 \\
\hline $\begin{array}{c}\text { Abstand } \\
1000\end{array}$ & 7 & 77,8 & 1 & 11,1 & 1 & 11,1 & 9 \\
\hline
\end{tabular}

Tab. 18: Zustand der Descemet-Membran; Anzahl und relative Häufigkeit in \%; Gruppe A (Abstand Bowman-Lamelle zu horizontalem Anteil des Lentikels); $(\mathrm{n}=54)$

In drei von sechs Gruppen ist analog zur Gesamtstatistik die „Minimalläsion“ am häufigsten. In der Gruppe „Abstand 650“ findet sich dreimal eine beidseitige Perforation, in der Gruppe „Abstand 750“ einmal.

Bei der weiteren Auswertung fällt auf, dass in der Gruppe „Abstand 1000“ mit 77,8 \% der Fälle, in der Gruppe „Abstand 950“ mit 71,4\% der Fälle die Membran im Vergleich zu den übrigen Gruppen mehr als doppelt so häufig intakt ist und sich auch keine beidseitige Perforation findet. 


\section{Zustand der Descemet-Membran in Gruppe C}

Zur Überprüfung der Vermutung, dass mit abnehmender Lentikeldicke die Descemet-Membran häufiger intakt bleibt, wurde Gruppe C gebildet und ausgewertet.

\begin{tabular}{|c|c|c|c|c|c|c|c|}
\hline & \multicolumn{2}{|c|}{$\begin{array}{c}\text { beide Seiten } \\
\text { intakt }\end{array}$} & \multicolumn{2}{|c|}{ Teilperforation } & \multicolumn{2}{|c|}{ Minimalläsion } & \multirow[t]{2}{*}{$\mathbf{n}$} \\
\hline & $\mathbf{n}$ & $\%$ & $\mathbf{n}$ & $\%$ & $\mathbf{n}$ & $\%$ & \\
\hline $\begin{array}{c}\text { Dicke } \\
\text { Lentikel } \\
931\end{array}$ & 3 & 23,1 & 3 & 23,1 & 7 & 53,8 & 13 \\
\hline $\begin{array}{c}\text { Dicke } \\
\text { Lentikel } \\
631\end{array}$ & 9 & 31,0 & 7 & 24,2 & 13 & 44,8 & 29 \\
\hline $\begin{array}{c}\text { Dicke } \\
\text { Lentikel } \\
330\end{array}$ & 8 & 66,7 & 1 & 8,3 & 3 & 25,0 & 12 \\
\hline
\end{tabular}

Tab. 19: Zustand der Descemet-Membran; Anzahl und relative Häufigkeit in \%; Gruppe C (Abstand Descemet-Membran zu horizontalem Anteil des Lentikels = Dicke des Lentikels); $(\mathrm{n}=54)$

Es fällt auf, dass in der Gruppe „Dicke Lentikel 330" in 66,7 \% der Fälle die Descemet-Membran intakt ist, d. h. mehr als doppelt so häufig wie im Vergleich zu den anderen beiden Gruppen.

\subsection{Abstand der nächstliegenden Blasen zur Descemet- Membran}

\section{Abstand in der Gesamtbetrachtung}

Es wurde auf beiden Seiten des Lentikels der Abstand der jeweils nächstliegenden Blase zur Descemet-Membran gemessen. 


\begin{tabular}{|c|c|c|c|c|c|c|}
\hline$[\mu \mathrm{m}]$ & Min & Max & MW & SD & MD & n \\
\hline $\begin{array}{c}\text { vertikal } \\
\text { rechts }\end{array}$ & 0 & 400 & 113,4 & 113,2 & 80,0 & 54 \\
\hline $\begin{array}{c}\text { vertikal } \\
\text { links }\end{array}$ & 0 & 850 & 113,8 & 141,7 & 72,5 & 54 \\
\hline
\end{tabular}

Tab. 20: Minimum, Maximum, Mittelwert, Standardabweichung \& Median des Abstandes der nächstliegenden Blasen zur Descemet-Membran; Gesamtstatistik; $(\mathrm{n}=54)$

Es kam dreimal auf der rechten sowie zweimal auf der linken Seite vor, dass die nächstliegende Blase direkt an der Descemet-Membran lag, wodurch der Minimumabstand von null zu erklären ist (s. Abb. 8). Der Maximumabstand links ist damit zu erklären, dass in einer Histologie auf der linken vertikalen Seite keine Blase entstanden ist, der Abstand also zur horizontalen Blasenlinie gemessen wurde (s. Abb. 9).

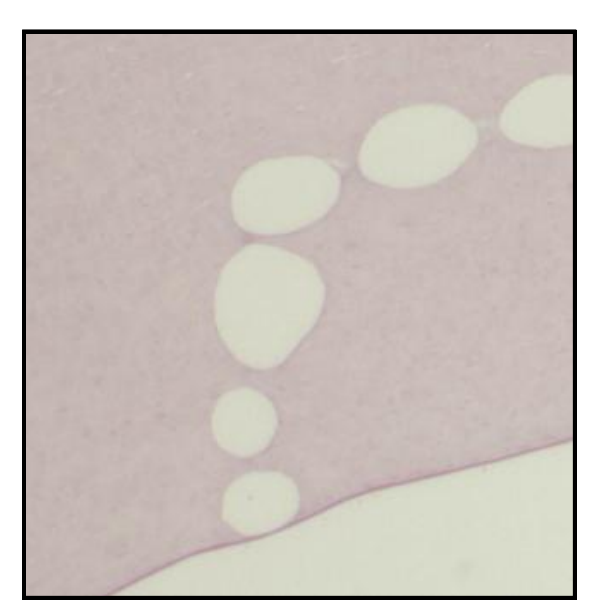

Abb. 8: direkt anliegende Blase links (x 5)

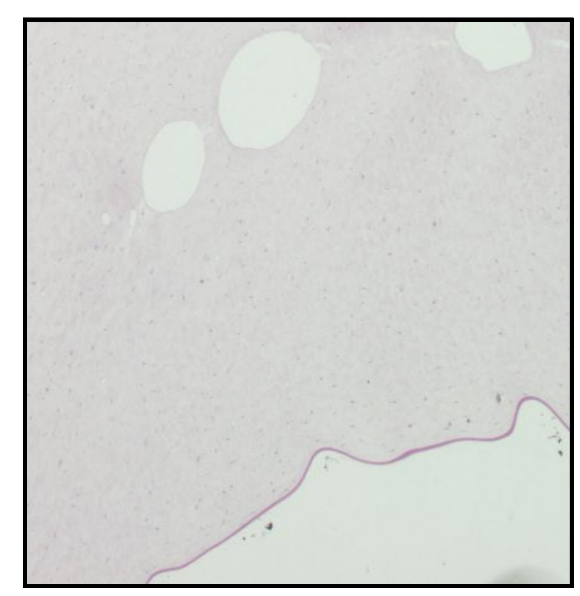

Abb. 9: fehlende Blase links (x 5)

Auffallend sind die nahezu gleichen Abstände auf beiden Seiten mit einer ähnlichen Standardabweichung.

Auch hier wurde der Wilcoxon-Test zur Prüfung der Unterschiede der beiden Seiten auf der Ebene der Gesamtstichprobe verwendet. 
Die Unterschiede zwischen den beiden Seiten sind mit $p=0,822$ nicht signifikant.

\section{Abstand in Gruppe A}

In Gruppe A ist die Verteilung wie folgt:

\begin{tabular}{|c|c|c|c|c|c|c|}
\hline$[\mu \mathrm{m}]$ & Min & Max & MW & SD & MD & $\mathbf{n}$ \\
\hline $\begin{array}{c}\text { Abstand } \\
550\end{array}$ & 5,0 & 210,0 & 67,2 & 67,8 & 45,0 & 9 \\
\hline $\begin{array}{c}\text { Abstand } \\
650\end{array}$ & 20,0 & 175,0 & 72,5 & 55,6 & 57,5 & 10 \\
\hline $\begin{array}{c}\text { Abstand } \\
750\end{array}$ & 0,0 & 380,0 & 95,0 & 123,5 & 40,0 & 9 \\
\hline $\begin{array}{c}\text { Abstand } \\
850\end{array}$ & 0,0 & 210,0 & 39,0 & 63,9 & 12,5 & 10 \\
\hline $\begin{array}{c}\text { Abstand } \\
950\end{array}$ & 145,0 & 850,0 & 285,7 & 251,8 & 210,0 & 7 \\
\hline $\begin{array}{c}\text { Abstand } \\
1000\end{array}$ & 10,0 & 395,0 & 174,4 & 125,0 & 165,0 & 9 \\
\hline
\end{tabular}

Tab. 21: Minimum, Maximum, Mittelwert, Standardabweichung \& Median des Abstandes der nächstliegenden Blasen zur Descemet-Membran vertikal links; Gruppe A (Abstand Bowman-Lamelle zu horizontalem Anteil des Lentikels); $(\mathrm{n}=54)$ 


\begin{tabular}{|c|c|c|c|c|c|c|}
\hline$[\mu \mathrm{m}]$ & Min & Max & MW & SD & MD & $\mathbf{n}$ \\
\hline $\begin{array}{c}\text { Abstand } \\
550\end{array}$ & 5,0 & 220,0 & 63,3 & 71,2 & 30,0 & 9 \\
\hline $\begin{array}{c}\text { Abstand } \\
650\end{array}$ & 0,0 & 400,0 & 126,5 & 147,8 & 75,0 & 10 \\
\hline $\begin{array}{c}\text { Abstand } \\
750\end{array}$ & 5,0 & 255,0 & 75,6 & 88,7 & 30,0 & 9 \\
\hline $\begin{array}{c}\text { Abstand } \\
850\end{array}$ & 5,0 & 400,0 & 97,0 & 118,0 & 57,5 & 10 \\
\hline $\begin{array}{c}\text { Abstand } \\
950\end{array}$ & 25,0 & 370,0 & 190,7 & 118,9 & 215,0 & 7 \\
\hline $\begin{array}{c}\text { Abstand } \\
1000\end{array}$ & 0,0 & 310,0 & 145,0 & 98,9 & 115,0 & 9 \\
\hline
\end{tabular}

Tab. 22: Minimum, Maximum, Mittelwert, Standardabweichung \& Median des Abstandes der nächstliegenden Blasen zur Descemet-Membran vertikal rechts; Gruppe A (Abstand Bowman-Lamelle zu horizontalem Anteil des Lentikels); $(n=54)$

Analog zu oben wurde nun dieselbe Auswertung durchgeführt, d. h. der Abstand der jeweils nächstliegenden Blase zur Descemet-Membran der beiden vertikalen Seiten in den jeweiligen Abstandsstufen verglichen. Hierbei zeigen sich für alle sechs Stufen bei relativ kleinen Fallzahlen ( $\mathrm{n}$ zwischen sieben und zehn) ebenfalls in allen Fällen nicht signifikante Ergebnisse ( $p$ zwischen 0,176 und 0,888).

Insgesamt bestätigt sich also auch in Gruppe A, dass es keinen statistisch signifikanten Unterschied des Abstandes auf beiden vertikalen Seiten gibt.

\section{Zusammenhang intendierter Abstand - Abstand der nächstliegenden Blasen von der Descemet-Membran}

Mit zunehmendem intendierten Abstand von der Bowman-Lamelle fällt ein vergrößerter Abstand der beiden nächstliegenden Blasen zur DescemetMembran auf.

Zur Überprüfung dieser Tatsache wurden zunächst für beide Seiten jeweils die Korrelationen zwischen dem intendierten Abstand der Bowman-Lamelle 
und den Abständen der nächstliegenden Blasen zur Descemet-Membran berechnet. Nur wenn sich über alle intendierten Abstandsstufen hinweg ein solcher Trend nachweisen lässt, dass mit größeren intendierten Abstandsstufen der Bowman-Lamelle auch die Abstände zur DescemetMembran steigen, ist ein Einzelvergleich der Stufen sinnvoll.

In beiden Fällen zeigen sich hier zwar nur schwache bis mittlere positive Korrelationen in einer Höhe von $r=0,293$ (rechts) bzw. $r=0,331$ (links), die jedoch auf dem $5 \%$-Niveau signifikant sind bei einem $n$ von 54 Fällen (rechts:

$p=0,032$, links: $p=0,014)$. Es ist also wie vermutet ein Trend nachweisbar.

Daher wurden zur weiteren Aufklärung die beiden höchsten intendierten mit den beiden geringsten intendierten Abstandsstufen („Abstand 1000“ mit „Abstand 550" und „Abstand 950“ mit „Abstand 650") verglichen (MannWhitney-U-Test).

Der Vergleich der beiden Extremgruppen 1000 und 550 im intendierten Abstand der Bowman-Lamelle ergibt bei insgesamt $n=18$ Fällen in beiden Fällen ganz knapp nicht signifikante Ergebnisse mit $p=0,063$ rechts und $p=0,0503$ links.

Beim Vergleich der Abstände 950 zu 650 zeigt sich dagegen bei $n=17$ Fällen der Unterschied mit $p=0,001$ höchst signifikant und auf dem $1 \%$-Niveau abgesichert. Der nachgewiesene Tendenzunterschied geht hier in die Richtung, dass bei dem höheren intendierten Abstand zur BowmanLamelle auch die Abstände zur Descemet-Membran höher sind.

\section{Zusammenhang Lentikeldicke - Abstand der nächstliegenden Blasen von der Descemet-Membran}

Um diese Tendenz weiter zu überprüfen, wurde die Gruppe C gebildet und ausgewertet. Hierbei wurde angenommen, dass bei nahezu gleich dicken Corneae mit zunehmendem Abstand von der Bowman-Lamelle die Lentikeldicke geringer wird. 


\begin{tabular}{|c|c|c|c|c|c|c|}
\hline$[\mu \mathrm{m}]$ & Min & Max & MW & SD & MD & n \\
\hline $\begin{array}{c}\text { Dicke } \\
\text { Lentikel } \\
931\end{array}$ & 5 & 210 & 84,2 & 65,3 & 70,0 & 13 \\
\hline $\begin{array}{c}\text { Dicke } \\
\text { Lentikel } \\
631\end{array}$ & 0 & 850 & 102,8 & 172,3 & 35,0 & 29 \\
\hline $\begin{array}{c}\text { Dicke } \\
\text { Lentikel } \\
330\end{array}$ & 10 & 395 & 172,5 & 107,1 & 160,0 & 12 \\
\hline
\end{tabular}

Tab. 23: Minimum, Maximum, Mittelwert, Standardabweichung \& Median des Abstandes der nächstliegenden Blasen zur Descemet-Membran vertikal links Gruppe C (Abstand Descemet-Membran zu horizontalem Anteil des Lentikels = Dicke des Lentikels); $(\mathrm{n}=54)$

\begin{tabular}{|c|c|c|c|c|c|c|}
\hline$[\mu \mathrm{m}]$ & Min & Max & MW & SD & MD & n \\
\hline $\begin{array}{c}\text { Dicke } \\
\text { Lentikel } \\
931\end{array}$ & 0 & 400 & 78,1 & 116,4 & 30,0 & 13 \\
\hline $\begin{array}{c}\text { Dicke } \\
\text { Lentikel } \\
631\end{array}$ & 0 & 400 & 121,2 & 121,9 & 75,0 & 29 \\
$\begin{array}{c}\text { Dicke } \\
\text { Lentikel } \\
330\end{array}$ & 0 & 265 & 132,9 & 84,2 & 117,5 & 12 \\
\hline
\end{tabular}

Tab. 24: Minimum, Maximum, Mittelwert, Standardabweichung \& Median des Abstandes der nächstliegenden Blasen zur Descemet-Membran vertikal rechts Gruppe C (Abstand Descemet-Membran zu horizontalem Anteil des Lentikels = Dicke des Lentikels); $(\mathrm{n}=54)$

Auffallend sind auch in dieser Gruppe die höheren Abstandsmittelwerte in der Untergruppe „Dicke Lentikel 330“ mit 172,5 bzw. 132,9 $\mu \mathrm{m}$.

Es wurde zunächst geprüft, ob überhaupt eine signifikante Korrelation zwischen den Abständen zur Descemet-Membran und den drei Lentikeldickestufen existiert. Links ergibt sich eine Korrelation (SpearmanKoeffizient) von $r=-0,226$, rechts von $r=-0,257$. In beiden Fällen ist die Korrelation also schwach negativ, d. h., mit zunehmender Lentikeldicke 
werden die Mittelwerte kleiner. Allerdings sind beide Korrelationen mit $p=0,061$ links und $p=0,101$ rechts nicht signifikant, es könnte sich also noch um einen zufälligen Zusammenhang handeln. Da die Signifikanz aber jeweils nur knapp verfehlt wird, kann man durchaus annehmen, es gäbe eine solche Beziehung in der Grundgesamtheit - nur lässt sie sich in der vorliegenden Stichprobe nicht genügend sicher gegen Zufall absichern.

Da die Signifikanz der Korrelationen hier jeweils nur sehr knapp verpasst wird, wurden trotz der nicht signifikanten Ergebnisse noch die beiden Extremgruppen, die größte und die kleinste Lentikeldickestufe („Dicke Lentikel 330“ und „Dicke Lentikel 931“) miteinander verglichen.

Die U-Tests zeigen hier rechts ein ebenfalls knapp nicht signifikantes Ergebnis mit $p=0,068$ und links einen auf dem $5 \%$-Niveau signifikanten Unterschied mit $p=0,030$. Wenn man also die beiden Extremgruppen der drei Stufen vergleicht, ist zumindest links ein Unterschied nachweisbar.

\subsection{Zusammenhang Abstand der nächstliegenden Blasen von der Descemet-Membran - Zustand der Membran}

Es wurden die Mittelwerte des Abstandes der nächstliegenden Blasen von der Descemet-Membran für die Gruppe B berechnet:

\begin{tabular}{|c|c|c|c|c|c|c|}
\hline$[\mu \mathrm{m}]$ & Min & Max & MW & SD & MD & $\mathbf{n}$ \\
\hline $\begin{array}{c}\text { beide Seiten } \\
\text { intakt }\end{array}$ & 5,0 & 850,0 & 188,5 & 185,6 & 165,0 & 20 \\
\hline Teilperforation & 0,0 & 150,0 & 44,5 & 48,5 & 25,0 & 11 \\
\hline Minimalläsion & 5,0 & 380,0 & 81,9 & 95,1 & 40,0 & 23 \\
\hline
\end{tabular}

Tab. 25: Minimum, Maximum, Mittelwert, Standardabweichung \& Median des Abstandes der nächstliegenden Blasen zur Descemet-Membran vertikal links; Gruppe B (Zustand der Descemet-Membran); $(\mathrm{n}=54)$ 


\begin{tabular}{|c|c|c|c|c|c|c|}
\hline$[\mu \mathrm{m}]$ & Min & Max & MW & SD & MD & n \\
\hline $\begin{array}{c}\text { beide Seiten } \\
\text { intakt }\end{array}$ & 5,0 & 400,0 & 184,5 & 114,1 & 142,5 & 20 \\
\hline Teilperforation & 0,0 & 185,0 & 76,8 & 67,9 & 60,0 & 11 \\
\hline Minimalläsion & 0,0 & 400,0 & 69,1 & 101,1 & 30,0 & 23 \\
\hline
\end{tabular}

Tab. 26: Minimum, Maximum, Mittelwert, Standardabweichung \& Median des Abstandes der nächstliegenden Blasen zur Descemet-Membran vertikal rechts; Gruppe B (Zustand der Descemet-Membran), $(\mathrm{n}=54)$

Im Anschluss wurden die Untergruppe „beide Seiten intakt“ mit den Untergruppen „Teilperforation“ bzw. „Minimalläsion“ verglichen (MannWhitney U-Test).

Beim Vergleich zur „Teilperforation“ zeigt sich eine Signifikanz von $p=0,002$ für links, bzw. $p=0,009$ für rechts.

Beim Vergleich zur „Minimalläsion“ ist die Signifikanz $p=0,006$ für links, bzw. $p=0,001$ für rechts.

Damit ist nachgewiesen, dass bei der Gruppe „beide Seiten intakt“ die Abstände der beiden nächstliegenden Blasen größer sind als jeweils bei den Gruppen „Teilperforation“ oder „Minimalläsion“. 


\section{Diskussion}

Zusammenfassend lassen sich die eingangs gestellten Fragen wie folgt beantworten:

\subsection{Abstand der Bowman-Lamelle zur horizontalen Blasenlinie}

Mit Ausnahme der Gruppe „Abstand 1000" besteht im Durchschnitt ein negativer Unterschied - d. h., der gemessene Abstand ist geringer als der zuvor eingestellte - zwischen 115-160 $\mu \mathrm{m}$. Dieser ist in allen Gruppen relativ konstant, so dass hier davon ausgegangen werden kann, dass die Corneae durch ihre Fixierung in Paraformaldehydlösung geschrumpft sind. Dieser Vorgang der Dehydratation ist ein homogener Vorgang, der die ganze Cornea gleichmäßig betrifft [63].

Der Laser arbeitet also bis auf 115-160 $\mu \mathrm{m}$ abzüglich dieses „Schrumpfungsfaktors“ präzise. Um die genaue Abweichung festzustellen, könnte man in zukünftigen Studien die Corneadicke vor der Laserprozedur z. B. mittels Pachymetrie bestimmen, um im Vergleich mit der histologischen Messung zu sehen, um wie viel sie durch die histologische Aufarbeitung schrumpft.

Eine weitere Möglichkeit wäre die Nutzung der „optical coherence tomography" (OCT) mit der man noch in vivo die entstehenden Lentikel vermessen kann. Hierbei treten keine Veränderungen der Cornea durch die histologische Aufarbeitung auf. Zudem fällt - im Gegensatz zur Pachymetrie - die Kontaktmessung, bei der es zu Eindellungen der Corneaoberfläche kommen kann, weg [26, 64].

\section{Abstand der Bowman-Lamelle zur horizontalen Blasenlinie im Vergleich mit weiteren Studien}

Für fünf Untergruppen der Gruppe A kann gezeigt werden, dass der Laser den gewünschten Abstand „Bowman-Lamelle zu entstehender horizontaler 
Blasenlinie" gleichmäßig über die gesamte Länge - gemessen jeweils an drei verschiedenen Punkten - des entstehenden Lentikels reproduzierbar und präzise erzeugt. Im Gegensatz hierzu ergeben die Messungen für Abstand 1000 einen deutlich signifikanten Unterschied.

Für die Streuungen dieses Abstandes kann bei zwei („oben“, „mittig“) der drei Messpunkte eine Gleichheit gezeigt werden. Für „unten“ ist ein Streuungsunterschied ebenfalls nicht nachgewiesen, kann aber auch nicht ausgeschlossen werden, da die Signifikanz nicht deutlich verfehlt wird.

In den Stichprobendaten gibt es einen Trend, dass mit zunehmendem Abstand auch die Spannweiten steigen. Dieser speist sich vor allem aus den auffallend hohen Spannweiten in der Untergruppe Abstand 1000. Die Vermutung lag daher nahe, dass tendenziell die Spannweiten steigen, wenn der intendierte Abstand größer wird. Trotz einer hohen Korrelation von 0,600 zwischen intendiertem Abstand und Spannweite bei der Betrachtung aller sechs Stufen ist diese nicht signifikant $(p=0,208)$. Es kann also nicht exakt nachgewiesen werden, dass eine abnehmende Präzision bei der Erzeugung von dünnen Lentikeln mit einer steigenden Spannweite existiert.

Bei den beiden letztgenannten untersuchten Parametern „intendierter Abstand" und "Spannweite" sind in der Untergruppe Abstand 1000 teils signifikante Unterschiede sowie Trends zu Unterschieden und damit Abweichungen bzw. abnehmende Präzision im Gegensatz zu den anderen Untergruppen festzustellen. Geht man von annähernd gleich dicken Corneae aus, sind diese Unterschiede also gerade bei der Erzeugung von dünnen Lentikeln zu erwarten. Ein Grund hierfür könnte im Aufbau der Cornea liegen, da die Cornea im anterioren Teil kompakter als im posterioren ist [51].

Gerade für operative Verfahren, bei denen exakt das krankhafte Gewebe entfernen werden muss und dementsprechend präzise dünne Lentikel benötigt werden, bedeutet dies eine Einschränkung der Genauigkeit und kann ein Nachteil sein.

VOSSMERBÄUMER et al. [63] bestätigen das Ergebnis einer gleichmäßigen Lentikelentstehung bei nahezu analoger Vorgehensweise (20/10 Laser, Formalinfixierung, Messung mittels Messokular) - allerdings bei der Produktion von LASIK-Flaps. Sie fanden in humanen Corneae, dass sich die Flapdicke in der Gesamtbetrachtung nicht statistisch signifikant zwischen der 
Peripherie $(189,0 \pm 71,7 \mu \mathrm{m})$ und dem Zentrum $(198,0 \pm 80.2 \mu \mathrm{m})$ des entstandenen Flaps unterscheiden.

Auch ALIÓ konnte mit Hilfe eines Hoch-Frequenz-Augen-Scanners (Artemis 2) zeigen, dass postoperativ die LASIK-Flaps homogen sind und ein nahezu planes Profil bei einer durchgehenden mittleren Dicke von $116 \mu \mathrm{m}$ (intendiert $110 \mu \mathrm{m}$ ) aufweisen [1].

BINDER et al. konstatierten, dass der produzierte LASIK-Flap bei einer intendierten Dicke von $110 \mu \mathrm{m}$ ca. 5-10 $\mu \mathrm{m}$ dünner ist als vorgesehen. Sie führen dies auf die Gewebeschrumpfung durch Fixation zurück [6]. Zudem vermuten sie, dass der Vorteil einer geringeren Spot- bzw. Line-Separation und daher reduzierten Rasterenergie möglicherweise darin liegt, die Abweichung der produzierten Flapdicke zu reduzieren. VOSSMERBÄUMER et al. hatten zwei verschiedene intendierte Flapdicken eingestellt: $140 \mu \mathrm{m}$ $(133,7 \pm 15,3)$ und $260 \mu \mathrm{m}(268,5 \pm 20,6)$. Ein „Schrumpfungsfaktor" wurde bewusst außer Acht gelassen. Wie in der vorliegenden Arbeit liegen auch hier die Standardabweichungen nahe beieinander und zeigen kaum einen Unterschied [63].

Im Gegensatz hierzu stellten JONES et al. in einem Vergleich des Femtosekundenlasers mit einem mechanischen Mikrokeratom bei einer tiefen lamellären Keratektomie fest, dass mit steigender intendierter Flapdicke die Standardabweichung höher ausfällt. Als Ursache hierfür wird von innen ein Verlust der Lasereffizienz bei tiefer Keratotomie vermutet [22].

Diese steigende Standardabweichung wird in der vorliegenden Studie zwar vermutet, kann allerdings statistisch nicht exakt nachgewiesen werden, so dass keine Aussage über die geringere Lasereffizienz getroffen werden kann.

In einer weiteren Studie - wiederum zu LASIK-Flaps - konnte BINDER ebenfalls steigende Standardabweichungen bei höherer Lentikeldicke zeigen (intendierte Dicke: $110 \mu \mathrm{m}$, Ergebnis: $125 \pm 12 \mu \mathrm{m}$; $140 \mu \mathrm{m}$, Ergebnis: 132,5 $\pm 18,5 \mu \mathrm{m})$. Diese Ergebnisse führt er allerdings auf die im Laufe der Studie gewonnenen Erfahrungen zurück, da er anfangs dickere Lentikel laserte [5]. Im Folgenden sollen nun einige Arbeiten vorgestellt werden, in denen ebenfalls die Lentikeldicke gemessen wurde. Teilweise wurde dabei eine 
ähnliche Vorgehensweise wie in der vorliegenden Arbeit angewandt oder der Femtosekundenlaser zur LASIK-Prozedur verwendet.

SOONG et al. führten bei menschlichen Corneae eine posteriore lamelläre Keratoplastik durch. Vom anterioren Epithel ausgehend begannen sie, zwischen 150-300 $\mu \mathrm{m}$ Tiefe zu lasern. Der entstandene Lentikel wurde zwischen zwei Deckgläser gelegt und die Dicke digital gemessen. Hierbei zeigte sich, dass der entstandene Lentikel $55 \pm 61 \mu \mathrm{m}$ dicker war als vor der Laserprozedur berechnet. Eine mögliche Ursache sehen die Autoren darin, dass vor der Prozedur zentral via Pachymetrie gemessen und kalkuliert wurde und die Messung zwischen den Deckgläsern die dickere periphere Cornea miteinbezog [51].

In weiteren Studien wurden LASIK-Flaps mittels eines Mikrometers gemessen. Hierbei trat eine maximale Abweichung zwischen 12 [44] und $24 \mu \mathrm{m}$ [19] bzw. bei intendierter Dicke von $180 \mu \mathrm{m}$ von 181,0 \pm 4,1 $\mu \mathrm{m}$ [29] auf. HOLZER et al. schränken ihre Aussagen allerdings dahingehend ein, dass die Lentikel möglicherweise zu dünn gemessen wurden durch den Druck, der auf die Deckgläser während der Messung ausgeübt wurde [19].

Bei KEZIRIAN et al. ist die erreichte Flapdicke etwas geringer $(114 \pm 14)$ als intendiert $(130 \mu \mathrm{m})$ [25], bei TALAMO et al. etwas größer (intendiert $110 \mu \mathrm{m}$, erreicht $119 \pm 12 \mu \mathrm{m}$ ) [54]. In allen Fällen erfolgten die Messungen jeweils mittels Pachymetrie direkt nach Anheben des entstandenen Flaps. Die Autoren konnten nachweisen, dass der Femtosekundenlaser statistisch gesehen besser reproduzierbare Flapdicken mit geringeren Standardabweichungen erzeugt als mechanische Mikrokeratome.

In einer klinischen Studie, bei der postoperativ mittels Hoch-FrequenzAugen-Scanner (Artemis 2) die LASIK-Flaps $(110 \mu \mathrm{m})$ beurteilt wurden, trat eine Standardabweichung von nur $\pm 6-7 \mu \mathrm{m}$ auf [1].

SUWAN APICHON et al. haben ein mögliches Transplantationsszenario entwickelt. Hierbei werden die Lamellen als Spendergewebe mittels des Lasers geschnitten (350 $\mu \mathrm{m}$ gemessen von der cornealen Oberfläche nach Entfernung des Epithels), im Anschluss für zwei Tage in Optisol-Lösung aufbewahrt und dann mittels Pachymetrie gemessen. Dabei zeigen sich Werte der anterioren Lamelle von 324,0 $\pm 112 \mu \mathrm{m}$ [53]. 
Bei JONES findet sich ein Wert von 400,0 $\pm 41 \mu \mathrm{m}$ bei intendierter tiefer lamellärer Keratektomie von $400 \mu \mathrm{m}$ [22]. In einer Studie von SARAYBA et al. wurden mittels eines Forschungsmodells des etablierten IntraLaseSystems ebenfalls anteriore Lamellen erzeugt $(300 \mu \mathrm{m}, 400 \mu \mathrm{m}, 500 \mu \mathrm{m})$ und mit einem digitalen Mikrometer direkt im Anschluss vermessen. Hierbei zeigten sich folgende Werte: 291,0 $\pm 5,0 \mu \mathrm{m}$ (intendiert 300), 385,0 $\pm 9,0 \mu \mathrm{m}$ (intendiert 400) und 488,0 $\pm 9,0 \mu \mathrm{m}$ (intendiert 500) [48].

In einer anderen Arbeit laserten die Autoren eine $200 \mu \mathrm{m}$ dicke Lamelle aus dem posterioren Stroma und entfernten diese über einen seitlich gelegenen Schnitt. Danach wurde diese Lamelle mittels eines Mikrokalipers vermessen, wobei die geringste Abweichung mit 0,67 $\pm 12,2 \mu \mathrm{m}$ angegeben wird [47].

Bisher existieren keine Untersuchungen, in denen ein ähnliches Vorgehen mit histologischer Beurteilung wie in der vorliegenden Arbeit gewählt wurde.

Daher ist eine Einordnung der gewonnenen Ergebnisse nur sehr schwer möglich. Zum einen verwenden die bisher publizierten Studien häufig unterschiedliche Methoden der Messung (Pachymetrie, digitales Mikrometer etc.) und damit andere Messmethoden als die vorliegende Arbeit. Zum anderen bezieht sich der Großteil auf Messungen von LASIK-Flaps. Diese beiden Aspekte erklären die breite Spannweite und Unterschiede in den Ergebnissen.

Für zukünftige Studien wäre besonders die Beurteilung eines auch klinisch praktikablen Vorgehens - wie z. B. das von SARAYBA et al. [47] - von Interesse.

Eine wünschenswerte technische Verbesserung wäre eine Resektion, die sich an den lamellären Strukturen orientiert und nicht eine konstante Schnitttiefe mit Bezug auf die anteriore Oberfläche der Cornea verwendet [48]. Technisch ist dieses allein mit dem Femtosekundenlaser bis dato allerdings nicht möglich. Eine mögliche Technik schildern Rocha und Durán [46] die eine lamelläre Dissektion mit Hilfe eines Nylon- bzw. Vicrylfadens durchführten. Hierbei erzielten sie eine gleichmäßige Tiefe der Dissektion mit einer homogenen stromalen Oberfläche. Diese Technik könnte man mit einer Femtosekundenlaser-Tunnelinzision wie bei der Implantation von Intacs (intrastromale corneale Ringsegmente) [14] verwenden, um die Vorteile beider Verfahren zu kombinieren. 


\subsection{Anzahl der Blasen}

In der horizontalen und vertikalen Ebene sind die Blasenanzahlen sowohl in der Gesamtbetrachtung als auch in den Untergruppen der Gruppe A nachweislich gleich.

Ebenso bestätigt sich die Annahme, dass die Blasenanzahl mit zunehmendem Abstand des Lentikels von der Bowman-Lamelle abnimmt.

\subsection{Länge und Breite der Blasen}

In der Gesamtbetrachtung lässt sich zeigen, dass der FEMTEC-Laser auf beiden vertikalen Seiten gleich lange und breite Blasen erzeugt.

In der Horizontalen sind die Blasen nachweislich länger als in der Vertikalen. Bezüglich der Breite ist dieser Unterschied zumindest im Vergleich zur rechten Seite signifikant, im Vergleich zur linken Seite wird die Signifikanz nur knapp verfehlt.

Der Durchschnitt der Breite der Blasen liegt bei 117,5-132,9 $\mu \mathrm{m}$. BINDER hingegen gibt den Durchmesser der Blasen - dieser entspricht der Breite der Blasen in der vorliegenden Arbeit - zwischen 25 und $75 \mu \mathrm{m}$ an, wobei zu berücksichtigen ist, dass keine Unterscheidung zwischen horizontaler und vertikaler Schnittebene getroffen wurde [6]. Die Fixierung der Corneae erfolgte, wie bei dieser Arbeit, ein bis zwei Minuten nach Ende der Laserprozedur. Die Pulsenergie des von BINDER verwendeten Lasers war allerdings mit 0,7 bzw. 0,4 $\mu \mathrm{J}$ deutlich niedriger als in der vorliegenden Studie, was eine Ursache für den Unterschied sein könnte.

Bezüglich Anzahl bzw. Länge und Breite der Blasen lässt sich also festhalten, dass der Femtosekundenlaser in der Lage ist, durch seine festgelegte Spotgröße und Spotseparation eine reproduzierbare Anzahl von Blasen gleicher Größe zu erzeugen und damit eine genaue Schnittführung ermöglicht. 


\subsection{Verhältnis von Blasen zu Gewebestegen}

Bzgl. des Verhältnisses „Blasenlänge zur Länge der Gewebestege“ kann bewiesen werden, dass in der horizontalen Ebene die Blasenlänge im Verhältnis zur Länge der dazwischen liegenden Gewebestege anteilig deutlich höher ist als in der vertikalen Ebene.

Eine sichere Aussage darüber, dass dieses Verhältnis auf beiden vertikalen Seiten gleich ist, ist nicht möglich. Zwar weisen die Ergebnisse der Gruppe A auf eine gleichmäßige Verteilung hin. In der Gesamtstatistik zeigt sich jedoch deskriptiv eine Tendenz, dass das Verhältnis rechts höher ist als links. Statistisch wird die Signifikanz dafür allerdings verfehlt. Es kann sich also durchaus noch um rein zufällige Unterschiede in der Stichprobe handeln.

Aufgabe zukünftiger Studien könnte sein, diese Tendenz auf Basis größerer Stichproben erneut zu überprüfen.

\subsection{Zustand des Gewebes und Verdichtungszonen}

Vom Verfasser wurde vermutet, dass der gezeigte höhere Anteil der Blasen an der horizontalen Schnittlinie auch einen Einfluss auf die Beschaffenheit der Gewebestege haben würde. Dies bestätigte sich nicht. Ebenso wenig konnte eine stärkere Einwirkung des Lasers auf die Gewebestege bei zunehmender Lentikeldicke und damit längerer vertikaler Seite nachgewiesen werden.

Zusammenhängende Blasen sowie Gewebebrücken werden bei BINDER et al. ähnlich beschrieben, wobei die Autoren den Eindruck hatten, dass mehr Gewebebrücken vorhanden waren, wenn die Spotseparation mit $7 \mu \mathrm{m}$ im Gegensatz zu $9 \mu \mathrm{m}$ geringer war. Unterschiede in der Gewebebeschaffenheit bzw. Muster der Gewebebrücken beschreiben die Autoren nicht [6].

In $88,9 \%$ der Fälle findet sich zumindest eine Verdichtungszone und in $57,4 \%$ davon eine zweite. Die Breite der Schäden ist hierbei bis zu 2,5 $\mu \mathrm{m}$ und damit deutlich breiter als bei LUBATSCHOWSKI, der das Ausmaß der thermischen Schäden mit ca. $1 \mu \mathrm{m}$ oder darunter - vergleichbar mit den 
Schäden, die durch die Excimer-Laser-Ablation entstehen würden - angibt [30]. Auch weitere Studien sprechen von geringeren Schäden, allerdings sind die Ergebnisse nur bedingt vergleichbar, da jeweils unterschiedliche Femtosekundenlaser verwendet wurden.

MAMON gibt an, dass zumindest elektronenmikroskopisch im Bereich der Schnittführung eine minimal thermisch bedingte Koagulation beobachtet werden kann [32]. Bei anderen zeigt sich mittels Transmissionselektronenmikroskop (TEM) in diesem Bereich sogar nur eine feine, elektronendichte Schicht (5-40 nm breit) mit angrenzenden normalen Kollagenfasern ohne Hinweise auf thermische Schäden [50].

MELTENDORF kann ebenso fast keine thermischen Veränderungen nach der histopathologischen Aufarbeitung feststellen, wobei er eine genaue Beschreibung dieser Schäden unterlässt [37]. Bei BINDER et al. und CHENG et al. sind die mit TEM begutachteten Grenzflächen der Blasen ebenfalls glatt, die angrenzenden Keratozyten unbeschädigt und ohne thermische Schäden $[5,9]$.

In anderen Studien ist die Beschreibung der Schäden nur vage: So sprechen beispielsweise SOONG et al. von einer "stuckartigen“ Struktur, welche unter dem Elektronenmikroskop zu sehen sei [51].

Eine genaue Unterscheidung zwischen durch Kompression bedingte Verdichtung des Gewebes bzw. thermische Schäden war in dieser Arbeit lichtmikroskopisch nicht durchzuführen.

\subsection{Zusammenhang Abstand der nächstliegenden Blasen von der Descemet-Membran - Zustand der Membran}

Die Abstandswerte der jeweils nächstliegenden Blase zur DescemetMembran zeigen beim Vergleich der beiden vertikalen Seiten sehr deutlich nicht signifikante Unterschiede, so dass in der Gesamtheit von einer Gleichheit des Abstandes der beiden nächstliegenden Blasen zur DescemetMembran auf beiden Seiten ausgegangen werden kann.

Damit zeigt sich, dass der Laser auf beiden Seiten gleich arbeitet und der Abstand der nächstliegenden Blasen zur Descemet-Membran reproduzierbar 
ist. In Gruppe A bestätigt sich diese Aussage. Lediglich in der Untergruppe „Abstand 950" können Unterschiede statistisch letztlich nicht ganz ausgeschlossen werden.

Des Weiteren kann zumindest ein Tendenzunterschied nachgewiesen werden, dass bei höherem intendiertem Abstand zur Bowman-Lamelle auch die Abstände der Blasen zur Descemet-Membran höher ausfallen.

Bezüglich des Zustandes der Membran wurde nachgewiesen, dass bei der Gruppe „beide Seiten intakt“ die Abstände der beiden nächstliegenden Blasen größer sind als jeweils in den Gruppen "Teilperforation“ oder „Minimalläsion“.

In den Berechnungen der Gruppe C wurde zudem die Vermutung gestärkt, dass bei geringerer Lentikeldicke der Abstand der beiden nächstliegenden Blasen höher ausfällt.

Fazit ist also: Mit abnehmender Lentikeldicke steigt sehr wahrscheinlich die Entfernung der beiden nächstliegenden Blasen zur Descemet-Membran und damit ist die Wahrscheinlichkeit für deren Perforation geringer.

Dieser Zusammenhang könnte genauer untersucht werden, indem man ein umgekehrtes Vorgehen wählt und die zu lasernde Lentikelhöhe einstellt (nach vorheriger Messung der Corneadicke mittels Pachymetrie), mit anschließender Überprüfung des Zustandes der Descemet-Membran.

In einer anderen Studie, die ebenfalls den FEMTEC-Laser verwendete und in der menschliche Corneae trepaniert wurden, zeigt sich hingegen, dass die Descemet-Membran nicht durchtrennt, sondern nur nach posterior gewölbt wird. Um eine gleichmäßige Durchtrennung der Membran zu erreichen, empfehlen die Autoren deshalb die Entwicklung eines spezifischen „Laserapplikationsmusters“ für den posterioren Teil der Cornea [37].

Die Frage, wie eine genaue Perforation der Descemet-Membran erreicht werden kann, ist von der Forschung noch zu beantworten. 


\subsection{Darstellung der Verfahren „Descemet Stripping Automated Endothelial Keratoplasty“ (DSAEK) und „Descemet Membrane Endothelial Keratoplasty“ (DMEK)}

Seit Einführung der PLAK sind weitere Verfahren entwickelt worden und befinden sich derzeit in der klinischen Erprobung. Bei der „Descemet Stripping Automated Endothelial Keratoplasty“ (DSAEK) wird die WirtsDescemet-Membran isoliert mittels „Descemet Stripping/Descemetorhexis“ entfernt [35,39]. Ein korneosklerales Scheibchen - welches als Spendergewebe dient - wird in eine künstliche Vorderkammer positioniert und fixiert. Dann werden entweder manuell, mit einem Mikrokeratom oder einem Femtosekundenlaser ca. 300-350 $\mu \mathrm{m}$ des vorderen Stromas entfernt. Das Reststroma wird auf einem Stanzblock ausgestanzt. Im Anschluss wird die posteriore Spenderlamelle manuell (dann „Descemet Stripping Endothelial Keratoplasty“ (DSEK) genannt) oder automatisiert mit dem Mikrokeratom oder dem Femtosekundenlaser [8] gewonnen (Dicke zwischen 100-200 $\mu \mathrm{m}$ ) und dann en bloc oder wie ein „Taco“ gefaltet durch eine schmale Inzision transplantiert und nahtfrei durch Injektion einer Luftblase in der Vorderkammer fixiert.

Ähnlich wie die PLAK eignet sich dieses Verfahren für Patienten mit endothelialen Corneaerkrankungen (z.B. Guttae) die zu einer reversiblen Trübung der Cornea durch stromales und/oder epitheliales Ödem geführt haben. Die DSAEK kann bei der Fuchsschen endothelialen Hornhautdystrophie auch mit einer Kataraktoperation im Sinne einer „neuen“ Triple-Operation kombiniert werden [10].

Eine häufig auftretende Komplikation ist die primäre inkomplette Adhärenz der posterioren Stromalamelle. Zudem kann es zu Interfacedebris (Verschleppung von Blut aus dem Korneoskleraltunnel der Inzision, Tupferfragmenten, Irispigment etc.) kommen. Zur Prophylaxe eines Pupillarblockwinkelblockglaukoms empfehlen manche Autoren eine prä- oder intraoperative Iridotomie [11].

Das neueste Verfahren ist die so genannte „Descemet Membrane Endothelial Keratoplasty“ (DMEK) nach MELLES et. al., bei dem nur die Descemet-Membran mit Endothel transplantiert wird [36]. Hierzu wird die 
Vorderkammer des Empfängers mit einer Luftblase gefüllt und im Anschluss mittels eines dafür entwickelten Spatels eine Descemetorhexis - d. h. die alleinige Lösung der Descemet-Membran vom Stroma - durchgeführt $[35,39]$. Das als Spendergewebe dienende korneosklerale Scheibchen wird auf $9 \mathrm{~mm}$ trepaniert und im Anschluss mittels einer Mikropinzette dessen Descemet-Membran vorsichtig abgezogen. Dabei entsteht spontan eine "Descemet-Membran-Rolle“, welche dann mittels eines Injektors durch eine schmale $(3,5 \mathrm{~mm})$ Inzision im Bereich des Limbus in die Vorderkammer platziert und dort mittels einer Luftblase für 30 Minuten fixiert wird.

MELLES sieht die Vorteile dieses Verfahrens im minimalen operativen Trauma (nur 3,5 mm Inzision im Gegensatz zu 4-5 mm Inzisionen bei der DSAEK) und in der zu erwartenden schnelleren und kompletteren visuellen Rehabilitation. Er geht davon aus, dass Probleme, die durch Dehydratation und Gewebeadaptation des transplantierten Gewebes bei der DSAEK entstehen, durch die geringere Menge transplantierten Materials wegfallen. Zudem erwartet er eine gute Langzeitüberlebensrate der Transplantate, da - ähnlich wie bei der DSAEK - eine nahezu komplette endotheliale Zellschicht verpflanzt werden kann. Ein weiterer Vorteil sei die mögliche Verwendung von vernarbten oder voroperierten Corneae als Spendergewebe, da nur die Descemet-Membran und das Endothel verwendet werden. Dies könne zu einer besseren Nutzung der limitierten Anzahl von Spendercorneae führen.

DAPENA et al. gehen zudem von einer besseren und einfacheren Verfügbarkeit des Spendergewebes für die DMEK aus, da die meisten Organspendebanken korneosklerale Scheibchen als Spendergewebe zur Verfügung stellen. Ihre Erfahrung zeige, dass es bisher bei der Bereitstellung von einzelnen Descemet-Membranen innerhalb Europas keine Probleme gegeben habe [12].

Mögliche Nachteile des Verfahrens liegen in der schwierigen operativen Handhabung, dem Zerreißen der Descemet-Membran während der Präparation sowie deren inkompletter postinterventioneller Adhärenz [43]. 


\subsection{Vergleich der klinischen Ergebnisse von PLAK, DSAEK und DMEK}

BAHAR et al. veröffentlichten 2008 eine große Vergleichsstudie zu DLEK, DSEK und DSAEK [2]. Die Unterschiede zwischen den Verfahren hinsichtlich der besten korrigierten Sehschärfe (best spectacle-corrected visual acuity = BSCVA) sind deutlich. Diese liegt bei der DLEK bei 20/80, bei der DSEK bereits bei 20/56 und bei der DSAEK schließlich bei 20/44. Auch bei der unkorrigierten Sehschärfe (uncorrected visual acuity = UCVA) ist eine Verbesserung der Werte von DLEK zu DSEK und DSAEK zu sehen.

Das sphärische Äquivalent ist fast gleich in allen Gruppen. Der Astigmatismus beträgt postinterventionell 1,61 \pm 1,26 Dioptrien (D) (DLEK), $1,86 \pm 1,1 \mathrm{D}(\mathrm{DSEK})$ und 1,36 \pm 0,92 D (DSAEK).

Die minimale Endothelzellzahl nach einem Jahr wird mit 1601,0 \pm 687,0 Zellen $/ \mathrm{mm}^{2}$ angegeben. Dies entspricht einem Zellverlust zwischen 36,4 \pm $15,2 \%$ und 43,4 $\pm 22,2 \%$. Es konnte kein signifikanter Unterschied zwischen den drei Verfahren festgestellt werden. Die höchste Dislokationsrate des Spendergewebes gab es in der DSAEK-Gruppe mit 15,6\%. Die Autoren führen dies auf die glattere Oberflächenstruktur und die noch geringe operative Erfahrung mit diesem Verfahren zurück.

Im Gegensatz hierzu attestieren TERRY und OUSLEY [60,40] der DLEK in einer Zwei-Jahres-Studie bessere Ergebnisse. Nach einem Jahr postoperativ beträgt die BSCVA 20/50, nach zwei Jahren 20/48 ohne signifikanten Unterschied. $90 \%$ der Patienten sehen besser als vor dem Eingriff. Die Endothelzellzahl ist mit 2335,0 \pm 468,0 Zellen $/ \mathrm{mm}^{2}$ (nach einem Jahr) bzw. $2151,0 \pm 457,0$ Zellen $/ \mathrm{mm}^{2}$ (nach zwei Jahren) ebenfalls höher. Dies entspricht einem signifikanten Endothelzellenverlust innerhalb des zweiten Jahres von $7,9 \%$.

Der Astigmatismus wird mit 2,04 \pm 1,05 D nach einem Jahr und mit 1,76 \pm 0,66 D nach zwei Jahren angegeben. Der aufgetretene Unterschied ist nicht signifikant.

Über einen Zeitraum von zwei Jahre hinweg konnten damit stabile, sehr gute Ergebnisse bzgl. des Visus und der Endothelzellzahl gezeigt werden. 
In einer weiteren Studie der gleichen Autoren finden sich sechs Monate nach DLEK ähnliche Ergebnisse mit einer durchschnittlichen BSCVA von 20/46 und einer Endothelzellzahl von 2140,0 $\pm 427,0$ Zellen $/ \mathrm{mm}^{2}$. Dies entspricht einem Endothelzellverlust von $23 \%$. Der Astigmatismus wurde mit 1,34 \pm $0,86 \mathrm{D}$ gemessen [40].

Im Gegensatz zu den Ergebnissen von BAHAR trat hier eine Dislokation des Spendergewebes nur in vier von 98 Fällen auf - bei diesen Patienten konnte die Lamelle mittels Injektion einer Luftblase wieder repositioniert werden.

Für die DSAEK berichtet GOROVOY in einer Arbeit, dass elf von sechzehn Patienten nach zwölf Wochen eine BSCVA von 20/40 hatten, nach einem Jahr hatten alle Patienten 20/40 oder besser [16].

Die durchschnittliche Endothelzellzahl liegt in seiner Studie nach zehn Monaten bei 1714 Zellen/ $/ \mathrm{mm}^{2}$, was einem Endothelzellverlust von $40 \%$ entspricht. Dieser Wert liegt damit höher als der, den TERRY für die DLEK angibt. Allerdings wurden seine Werte bereits sechs Monaten nach der Operation gemessen. GOROVOY führt seine geringeren Endothelzellzahlen daher auf die zeitlich bedingte weitere Abnahme zurück.

In $25 \%$ der Fälle kam es zu einer Teildislokation der Lamelle, welche ebenfalls durch erneute Injektion einer Luftblase behoben wurde. Eine totale Dislokation wurde nicht beobachtet.

GOROVOY sieht mögliche Ursachen für die Dislokation zum einen in den zwei sehr glatten Oberflächen, die bei der DSAEK entstehen, sowie der fehlenden „Empfängertasche“, die - anders als bei der DLEK - nicht vorhanden ist. Zudem vermutet er, dass bis dato unbekannte Faktoren des Stromas eine Rolle bei der Adhärenz der Spenderlamelle spielen und die Funktion des Endothels bei diesem Vorgang überschätzt wird. Einen Hauptvorteil der DSAEK sieht er darin, dass - anders als bei der PLAK - die schwierige Präparation der stromalen Lamelle wegfällt.

Für die Präparation der Spenderlamelle bei der DSEK kann auch ein Femtosekundenlaser verwendet werden (FS-DSEK). CHENG et al. verwendeten in ihrer Studie das IntraLase-System mit einer Pulsfrequenz von $30 \mathrm{kHz}$. Die BSCVA beträgt nach sechs Monaten im Mittel 20/57. 
Die durchschnittliche Endothelzellzahl ist $1368 \pm 425$ Zellen/mm². Dies entspricht $48 \%$ Zellverlust. Bei vier von 20 Patienten (20\%) trat eine Dislokation der transplantierten Lamelle auf.

Eine mögliche Erklärung für die im Vergleich schlechtere BSCVA sehen die Autoren in der Oberflächenbeschaffenheit des Interface. Durch den Femtosekundenlaser entsteht eine rauere Oberfläche. Eine weitere Erklärung könnte möglicherweise die Aktivierung von Keratozyten sein, die zu zunehmender Eintrübung („haze“) des Interface führen [8].

Den Autoren fiel auf, dass bei der FS-DSEK eine meniskusartig geformte Lamelle mit einer dickeren Peripherie entsteht. Sie geben zu bedenken, dass dies zu einer geringen hypermetropischen Verschiebung der Brechkraft führen kann („hyperopic shift“). Eine mögliche Lösung präsentieren IDE et al., die vermuten, dass bei der FS-DSEK und gleichzeitiger Verwendung von weichen, hypermetropischen Kontaktlinsen ein ebeneres Spendergewebe erzeugt werden kann. Die gemessenen Unterschiede sind allerdings nicht signifikant [20].

In einer Vergleichsstudie zwischen verschiedenen Pulsfrequenzen des IntraLase-Systems können CHENG et al. zeigen, dass durch FS-DSEK kein signifikanter Verlust von Endothelzellen im Vergleich zu nicht gelaserten Corneae der Kontrollgruppe entsteht. Der Endothelzellverlust liegt zwischen $4,3 \%(30 \mathrm{kHz})$ und $7,7 \%(15 \mathrm{kHz})$. Die Unterschiede zwischen den verschiedenen Pulsfrequenzen sind statistisch nicht signifikant.

Vorteil dieser FS-DSEK ist, dass die Spenderlamellen bis zu drei Wochen vor Transplantation aufbewahrt werden können. Dies ermöglicht z. B. serologische Untersuchungen sowie Antigentypisierung von Spender und Empfänger. Darüber hinaus können sie in einem automatisierten und standardisierten Prozess, z. B. in einer Hornhautbank, produziert und somit aus dem eigentlichen Operationsverlauf „ausgelagert" werden [7,9].

Bei der DMEK sind die veröffentlichten Ergebnisse noch besser. PRICE et al. geben an, dass bereits drei Monate nach DMEK die durchschnittliche BSCVA 20/25 beträgt, wobei insgesamt $94 \%$ der Patienten eine BSCVA von mindestens 20/40 haben [43].

HAM et al. beschreiben die gleiche BSCVA in ihrer ersten klinischen DMEKStudie [17]. Wie oben gezeigt, konnte bei den DSAEK-Studien erst nach 
sechs Monaten eine vergleichbare BSCVA gemessen werden [2,16]. PRICE et al. geben die Endothelzellzahl mit $3010 \pm 200$ Zellen $/ \mathrm{mm}^{2}$ an, der Endothelzellverlust beträgt $30 \pm 20 \%$ nach drei und $32 \pm 20 \%$ nach sechs Monaten. HAM gibt $28 \%$ Verlust an. Im Vergleich mit den genannten DSAEK- und DLEK-Studien zeigt sich hier ein geringerer Endothelzellverlust. In acht Prozent der Fälle kam es bei PRICE et al. zu einem Zerreißen der Descemet-Membran während der Descemetorhexis. Eine Konversion in eine DSAEK ist dann intraoperativ möglich, um den Verlust von Spendergewebe zu verhindern.

Bei mehr als der Hälfte der Patienten wurde die Anlage einer erneuten Luftblase nötig, da es zu Dislokationen der Descemet-Membran gekommen war. Die gleichen Operateure mussten dies bei der DSAEK in nur 3-4 \% der Fälle durchführen. Einen möglichen Grund sehen sie darin, dass sich bei der DMEK - im Gegensatz zur DSAEK - die partiell abgelöste DescemetMembran nicht wieder von alleine anlegt, sondern sich einrollt.

Damit können PRICE et al. zeigen, dass bei der DMEK schneller bessere visuelle Resultate mit einfacher zu berechnender postoperativer Brechkraft bei weniger Endothelzellverlust zu erzielen sind. Nachteile sind die schwierigen operativen Schritte mit häufigerer Reintervention. 


\section{Schlussfolgerungen}

Mit dem Femtosekundenlaser existiert ein präzises Instrument für die Durchführung der posterioren lamellären Keratoplastik. Die zuvor manuell durchgeführten operativ schwierigen Schritte mit geringer Reproduzierbarkeit der gewünschten Lamellendicken lassen sich - wie gezeigt - durch den Laser vereinfachen. $\mathrm{Er}$ ist in der Lage, die benötigten Lamellendicken gleichmäßig über die gesamte Breite des Lentikels zu produzieren. Er ist somit sowohl für die PLAK als auch DSEK gut nutzbar.

Da gezeigt werden konnte, dass der Femtosekundenlaser eine reproduzierbare Anzahl von Blasen gleicher Größe erzeugt, ist eine präzise Schnittührung möglich. Er kann daher auch für die Erzeugung anderer gewünschter Schnittmuster verwendet werden, um z. B. „selbstverschließende“" penetrierende Keratoplastiken ohne die Verwendung von Nahtmaterial zu ermöglichen [49].

In ca. $2 / 3$ der Fälle ist zumindest eine Läsion der Descemet-Membran nachweisbar, womit eine einfache Entfernung des entstandenen Lentikels möglich ist.

Ein Nachteil ist die abnehmende Wahrscheinlichkeit einer Läsion der Descemet-Membran bei geringerer Lentikeldicke sowie der Trend einer abnehmenden Präzision bei der Erzeugung von dünnen Lentikeln.

Da die PLAK bei Patienten mit endothelialen Hornhauterkrankungen eingesetzt wird, bei denen man nur exakt das krankhafte Gewebe entfernen will und dementsprechend präzise dünne Lentikel benötigt, bedeutet dies eine Einschränkung der Genauigkeit.

Künftige Studien sollten sich auf die Entwicklung eines standardisierten Verfahrens mit optimierten Einstellungen und Entwicklung eines spezifischen "Laserapplikationsmusters“ für den posterioren Teil der Cornea zur Erreichung der Ziele (verlässliche Lentikeldicke ohne Schwankungen, Perforation der Descemet-Membran) konzentrieren.

Ebenso wäre ein einheitliches Messverfahren zum Vergleich der erreichten Lentikeldicken wünschenswert, da Vergleiche mit weiteren Studien durch die Vielzahl an genutzten Messverfahren erschwert waren. Eine Möglichkeit wäre z. B. die Nutzung der „optical coherence tomography“ (OCT) mit der 
man noch in vivo die entstehenden Lentikel vermessen kann. Ein Vorteil dieses Verfahrens ist, dass hierbei keine Veränderungen der Cornea durch die histologische Aufarbeitung entstehen. Zudem fällt - im Gegensatz zur Pachymetrie - die Kontaktmessung, bei der es zu Eindellungen der Corneaoberfläche und damit ggf. einer Verfälschung des Messergebnisses kommen kann, weg.

Der Femtosekundenlaser verwendet eine konstante Schnitttiefe mit Bezug zur anterioren Oberfläche der Cornea. Um eine Resektion, die sich an den lamellären Strukturen orientiert, zu erreichen, könnte in Zukunft eine Kombination zweier Techniken verwendet werden. Zum einen die von Rocha et al. entwickelte Dissektionstechnik mit Hilfe eines Nylonfadens [46]. Zum anderen die etablierte Tunnelinzision mit Hilfe des Femtosekundenlasers wie bei der Implantation von cornealen Ringsegmenten.

Relevante thermische oder durch Kompression entstandene Schäden konnten in der vorliegenden Studie nicht gefunden werden. Genaue quantitative bzw. qualitative Aussagen zu solchen Schäden fehlen in den meisten anderen Studien. Eine zukünftige Studie könnte sich mit den genauen Ausdehnungen sowie Ursachen der in dieser Arbeit „Verdichtungszonen“ genannten Bereiche beschäftigen.

Neuere Studien weisen in die Richtung, dass die Weiterentwicklungen der PLAK - DSAEK und DMEK - bessere Ergebnisse liefern. Mit der FS-DSEK gibt es ein weiteres Gebiet, für das der Femtosekundenlaser sehr gut geeignet ist.

Es bleibt abzuwarten, ob die sich abzeichnende Überlegenheit der neueren Verfahren auch in Langzeitstudien bestätigt werden kann. 


\section{Literaturverzeichnis}

[1] Alió JL, Piñero DP: Very high-frequency digital ultrasound measurement of the LASIK flap thickness profile using the IntraLase femtosecond laser and M2 and Carriazo-Pendular microkeratomes. $J$ Refract Surg 2008; 24:12-23.

[2] Bahar I, Kaiserman I, McAllum P, Slomovic A, Rootman D: Comparison of posterior lamellar keratoplasty techniques to penetrating keratoplasty. Ophthalmology 2008; 115:1525-1533.

[3] Bamberger K: SPSS Grundlagen. RRZN Leibniz Universität Hannover 2006; 11:1-124.

[4] Benson WH, Goosey JD: Lamellar keratoplasty. Cornea 1997; 18331842.

[5] Binder P: Flap dimensions created with the IntraLase FS laser. J Cataract Refract Surg 2004; 30:26-32.

[6] Binder P, Sarayba M, Ignacio T, Juhasz T, Kurtz R: Characterization of submicrojoule femtosecond laser corneal tissue dissection. J Cataract Refract Surg 2008; 34:146-152.

[7] Cheng YY, Pels E, Cleutjens JP, van Suylen R, Hendrikse F, Nuijts RM: Corneal endothelial viability after femtosecond laser preparation of posterior lamellar discs for descemet-stripping endothelial keratoplasty. Cornea 2007; 26:1118-1122.

[8] Cheng YY, Hendrikse F, Pels E, Wijdh R, van Cleynenbreugel $H$, Eggink C, van Rij G, Rijneveld W, Nuijts RM: Preliminary results of femtosecond laser-assisted descemet stripping endothelial keratoplasty. Arch Ophthalmol 2008; 126:1351-1356. 
[9] Cheng YY, Kang SJ, Grossniklaus HE, Pels E, Duimel HJ, Frederik PM, Hendrikse F, Nuijts RM: Histologic evaluation of human posterior lamellar discs for femtosecond laser Descemet's stripping endothelial keratoplasty. Cornea 2009; 28:73-79.

[10] Covert DJ, Koenig SB: New triple procedure: Descemet's stripping and automated endothelial keratoplasty combined with phacoemulsification and intraocular lens implantation. Ophthalmology 2007; 114:1272-1277.

[11] Cursiefen C, Kruse FE: Posteriore lamelläre Keratoplastik (DSAEK). Ophthalmologe 2008; 105:183-192.

[12] Dapena I, Ham L, Melles GR: Endothelial keratoplasty: DSEK/DSAEK or DMEK - the thinner the better? Curr Opin Ophthalmol 2009; 20:299-307.

[13] Duncker G, Krumeich J, Wilhelm F, Bredehorn T: Lamellierende Keratoplastik - neue Konzepte. Klin Monatsbl Augenheilkd 2004; 221:14-23.

[14] Ertan A, Colin J: Intracorneal rings for keratoconus and keratectasia. $J$ Cataract Refract Surg 2007; 33:1303-1314.

[15] Eye Bank Association of America: Statistical Report. Washington D. C. 2002.

[16] Gorovoy MS: Descemet - Stripping automated endothelial keratoplasty. Cornea 2006; 25:886-889.

[17] Ham L, Dapena I, van Luijk C, van der Wees J, Melles G: Descemet membrane endothelial keratoplasty (DMEK) for Fuchs endothelial dystrophy: review of the first 50 consecutive cases. Eye 2009; 23:1990-1998.

[18] Hoffart L, Proust $H$, Matonti $F$, Catanèse $M$, Conrath $J$, Ridings $B$ : Kératoplastie lamellaire antérieure assistée par laser femtoseconde 
(Femtosecond-assisted anterior lamellar keratoplasty). J Fr Ophtalmol 2007; 30:689-694.

[19] Holzer M, Rabsilber T, Auffarth G: Femtosecond laser-assisted corneal flap cuts: morphology, accuracy and histopathology. IOVS 2006; 47:28282831.

[20] Ide T, Yoo SH, Kymionis GD, Leng T, Marini C, Stanciu NA, O'Brien TP: Descemet stripping automated endothelial keratoplasty tissue preparation with femtosecond laser and contact lens. Cornea 2010; 29:93-98.

[21] Jonas B: Corneal endothelial transplantation using femtosecond laser technology. Eye 2004; 18:657-658.

[22] Jones YJ, Goins KM, Sutphin JE, Mullins R, Skeie JM: Comparison of the femtosecond laser (IntraLase) versus manual microkeratome (Moria ALTK) in dissection of the donor in endothelial keratoplasty: initial study in eye bank eyes. Cornea 2008; 27:88-93.

[23] Juhasz T, Loesel H, Kurtz R, Horvath C, Bille J, Mourou G: Corneal refractive surgery with femtosecond laser. Journal of selected Topics in Quantum Electronics 1999; 5:902-910.

[24] Kaisermann I, Maresky HS, Bahar I, Rootman DS: Incidence, possible risk factors, and potential effects of an opaque bubble layer created by a femtosecond laser. J Cataract Refract Surg 2008; 34:417-423.

[25] Kezirian G, Stonecipher K: Comparison of the IntraLase femtosecond laser and mechanical keratomes for laser in situ keratomileusis. J Cataract Refract Surg 2004; 30:804-811.

[26] Kim JH, Lee D, Rhee KI: Flap thickness reproducibility in laser in situ keratomileusis with a femtosecond laser: optical coherence tomography measurement. J Cataract Refract Surg 2008; 34:132-136. 
[27] Koenig SB, Covert DJ, Dupps WJ, Meisler DM: Visual acuity, refractive error, and endothelial cell density six months after Descemet's stripping and automated endothelial keratoplasty (DSAEK). Cornea 2007; 26:670-674.

[28] Kurtz RM, Lie X, Elner VM: Photodisruption in the human cornea as a function of laser pulse width. $J$ Refract Surg 1997; 13:653-658.

[29] Loesel H, Zickler L, Kessler R: Refractive surgical applications of ultrashort pulse lasers. Aus: Bille J, Harner C, Loesel F (Hrgb.); AberrationFree Refractive Surgery. Springer Verlag Berlin 2004; 2-17.

[30] Lubatschowski H, Maatz G, Heisterkamp A, Hetzel U, Drommer W, Welling $\mathrm{H}$, Ertmer $\mathrm{W}$ : Application of ultrashort laser pulses for intrastromal refractive surgery. Graefe's Arch Clin Exp Ophthalmol 2000; 238:33-39.

[31] Lubatschowski $\mathrm{H}$ : Overview of commercially available femtosecond lasers in refractive surgery. J Refract Surg 2008; 24:102-107.

[32] Mamom T: Histologische und ultrastrukturelle Untersuchungen der Schweine- und Kaninchencornea nach intrastromaler Femtosekundenlaserbehandlung. These zur Erlangung eines Philosophical Doctors (PhD) im Fachgebiet Pathologie der tiermedizinischen Hochschule Hannover 2002.

[33] Melles G, Eggink F, Lander F: A surgical technique for posterior lamellar keratoplasty. Cornea 1998; 17:618-626.

[34] Melles G, Lander F, Van Dooren B, Pels E, Beekhuis W: Preliminary clinical results of posterior lamellar keratoplasty through a sclerocorneal pocket incision. Ophthalmology 2000; 107:1850-1856.

[35] Melles G, Wijdh RH, Nieuwendaal CP: A technique to excise the Descemet membrane from a recipient cornea (descemetorhexis). Cornea 2004; 23:286-288. 
[36] Melles G, San Ong T, Ververs B, van der Wees J: Descemet membrane endothelial keratoplasty (DMEK). Cornea 2006; 25:987-990.

[37] Meltendorf C, Schroeter J, Bug R, Kohnen T, Deller T: Corneal trephination with the femtosecond laser. Cornea 2006; 25:1090-1092.

[38] Morishige N, Kesler-Diaz A, Wahlert AJ, Kurtz RM, Juhasz T, Sarayba $\mathrm{M}$, Jester JV: Corneal response to femtosecond laser photodisruption in the rabbit. Exp Eye Res 2008; 86:835-843.

[39] Niewendaal CP, Lapid-Fortzak R, van der Meulen IJ, Melles G: Posterior lamellar keratoplasty using descemetorhexis and organ-cultured donor corneal tissue (Melles technique). Cornea 2006; 25:933-936.

[40] Ousley P, Terry MA: Stability of vision, topography, and endothelial cell density from 1 year to 2 years after deep lamellar endothelial keratoplasty surgery. Ophthalmology 2005; 112:50-57.

[41] Price MO, Price FW: Descemet's stripping with endothelial keratoplasty: comparative outcomes with microkeratome-dissected and manually dissected donor tissue. Ophthalmology 2006; 113:1936-1942.

[42] Price MO, Baig KM, Brubaker JW, Price FW: Randomized, prospective comparison of precut vs. surgeon-dissected grafts for Descemet's stripping automated endothelial keratoplasty. Am J Ophthalmol 2008; 146:36-41.

[43] Price MO, Giebel AW, Fairchild K, Price FW: Descemet's membrane endothelial keratoplasty. Ophthalmology 2009; 116:2361-2368.

[44] Ratkay-Traub I, Ferincz I, Juhasz T, Kurtz R, Krueger R: First clinical results with the Femtosecond-neodynium-glass laser in refractive surgery. J Refract Surg 2003; 19:94-103. 
[45] Ripken T, Bernau W, Oberheide U, Schumacher S, Rathjen C, Kermani O, Ziemer F, Lubatschowski $\mathrm{H}$ : DaVinci versus IntraLase and conventional microkeratomes for corneal lamellar cuts. Presented at the Annual Meeting "Building international collaborations" of the Association for Research in Vision and Ophthalmology. Ft. Lauderdale, FL, USA. April 30th-May 4th, 2006.

[46] Rocha GA, Durán JA, Acera A, Merayo J: Corneal lamellar dissection by using suture threads in porcine eyes. Cornea 2007; 26:1255-1257.

[47] Sarayba M, Juhasz T, Chuck R, Ignacio T, Nguyen T, Sweet P, Kurtz R: Femtosecond laser posterior lamellar keratoplasty. A Laboratory Model. Cornea 2005; 24:328-333.

[48] Sarayba M, Maguen E, Salz J, Rabinowitz Y, Ignacio T: Femtosecond laser keratome creation of partial thickness donor corneal buttons for lamellar keratoplasty. J Refract Surg 2007; 23:58-65.

[49] Seitz B, Langenbucher A, Hofmann-Rummelt C, Schlötzer-Schrehardt U, Naumann $\mathrm{GOH}$ : Nonmechanical posterior lamellar keratoplasty using the femtosecond laser (femto-PLAK) for corneal endothelial decompensation. Am J Ophthalmol 2003; 136:769-772.

[50] Seitz B, Brünner H, Viestenz A, Hofmann-Rummelt C, SchlötzerSchrehardt $U$, Langenbucher $A$ : Inverse mushroom-shaped nonmechanical penetrating keratoplasty using a femtosecond laser. Am J Ophthalmol 2005; 139:941-943.

[51] Soong H, Mian S, Abbasi O, Juhasz T: Femtosecond laser-assisted posterior lamellar keratoplasty - initial studies of surgical technique in eye bank eyes. Ophthalmology 2005; 112:44-49.

[52] Soong H, Malta J: Femtosecond lasers in ophthalmology. Am J Ophthalmol 2009; 147:189-197. 
[53] Suwan-Apichon O, Reyes J, Griffin N, Barker J, Gore P, Chuck R: Microkeratome versus femtosecond laser predissection of corneal grafts for anterior and posterior lamellar keratoplasty. Cornea 2006; 25:966-968.

[54] Talamo J, Meltzer J, Gardner J: Reproducibility of flap thickness with IntraLase FS and Moria LSK-1 and M2 microkeratomes. J Refract Surg 2006; 22:556-561.

[55] Terry M: The evolution of lamellar grafting techniques over 25 years. Cornea 2000; 19:611-616.

[56] Terry M, Ousley P: Endothelial replacement without surface corneal incision or sutures: topography of the deep lamellar endothelial keratoplasty procedure. Cornea 2001; 20:14-18.

[57] Terry M, Ousley P: Deep lamellar endothelial keratoplasty in the first United States patients: early clinical results. Cornea 2001; 20:239-243.

[58] Terry M, Ousley P: Replacing the endothelium without corneal surface incisions or sutures - the first US clinical series using the deep lamellar endothelial keratoplasty procedure. Ophthalmology 2003; 110:755-764.

[59] Terry M, Ousley P: Rapid visual rehabilitation after endothelial transplants with deep lamellar endothelial keratoplasty (DLEK). Cornea 2004; 23:143-153.

[60] Terry M, Ousley P: Deep lamellar endothelial keratoplasty. Visual acuity, astigmatism, and endothelial survival in a large prospective series. Ophthalmology 2005; 112:1541-1549.

[61] Terry M: Endothelial keratoplasty: history, current state and future directions. Cornea 2006; 25:873-878. 
[62] von Hippel A: Eine neue Methode der Hornhauttransplantation; Graefes Arch Ophthalmol 1888; 24:108-130.

[63] Vossmerbäumer U, Jonas JB: Regularity of human corneal flaps prepared by femtosecond laser technology. J Refract Surg 2008; 24:645-648.

[64] Yoo SH, Kymionis GD, Deobhakta AA, Ide T, Manns F, Culbertson WW, O'Brien TT, Alfonso EC: One year results and anterior segment optical coherence tomography (AS OCT) findings of descemet stripping automated endothelial keratoplasty (DSAEK) combined with phacoemulsification. Arch Ophthalmol 2008; 126:1052-1055. 


\section{Abbildungsverzeichnis}

Abb. 1-9 jeweils eigene Darstellung mit freundlicher Unterstützung von Fr. C. Hofmann-Rummelt.

\section{Abkürzungsverzeichnis}

\begin{tabular}{|c|c|}
\hline BSCVA & Best Spectacle-Corrected Visual Acuity \\
\hline bzgl. & bezüglich \\
\hline bzw. & beziehungsweise \\
\hline D & Dioptrie \\
\hline d. h. & das heißt \\
\hline DLEK & Deep Lamellar Endothelial Keratoplasty \\
\hline et al. & et alii \\
\hline etc. & et cetera \\
\hline ggf. & gegebenenfalls \\
\hline Hrgb. & Herausgeber \\
\hline Intacs & intrastromale corneale Ringsegmente \\
\hline Kap. & Kapitel \\
\hline LASIK & Laser in situ Keratomileusis \\
\hline PAS & Periodic Acid Schiff \\
\hline PLAK & posteriore lamelläre Keratoplastik \\
\hline S. & siehe \\
\hline UCVA & Uncorrected Visual Acuity \\
\hline USA & United States of America \\
\hline vgl. & vergleiche \\
\hline v. a. & vor allem \\
\hline z. B. & zum Beispiel \\
\hline
\end{tabular}




\section{Danksagung}

Es ist mir ein Anliegen, all jenen Personen zu danken, die zum Gelingen dieser Arbeit beigetragen haben.

Ich möchte mich bei meinem Doktorvater Herrn Professor Dr. med. B. Seitz für die freundliche Überlassung des Themas sowie kontinuierliche Unterstützung und Hilfestellung bedanken.

Herrn Prof. Dr. hum. biol. A. Langenbucher danke ich für die Hilfe bei der statistischen Auswertung sowie seine ausführlichen Korrekturen.

Mein besonderer Dank gilt Frau Hofmann-Rummelt für manch wertvollen Tipp gerade zu Beginn der Arbeit und ihre freundliche Unterstützung bei der histologischen Aufarbeitung und der Erstellung der Fotos.

Frau B. Omutz, Herrn Dr. Sör, Frau V. Fuchs und Frau D. Pörs danke ich für ihre Hilfe. 